\title{
The eclipsing binary star RZ Cas: accretion-driven variability of the multimode oscillation spectrum
}

\author{
D. E. Mkrtichian, ${ }^{1 \star}$ H. Lehmann, ${ }^{2}$ E. Rodríguez, ${ }^{3}$ E. Olson, ${ }^{4}$ S.-L. Kim, ${ }^{5}$ \\ A. V. Kusakin, ${ }^{6,7}$ J. W. Lee, ${ }^{5}$ J.-H. Youn, ${ }^{5}$ S.-G. Kwon, ${ }^{8}$ M. J. López-González, ${ }^{3}$ \\ E. Janiashvili, ${ }^{9}$ S. K. Tiwari, ${ }^{10}$ Santosh Joshi, ${ }^{11}$ P. Lampens, ${ }^{12}$ P. Van Cauteren, ${ }^{13}$ \\ L. Glazunova, ${ }^{14,15}$ A. Gamarova, ${ }^{15}$ K. N. Grankin, ${ }^{16}$ E. Rovithis-Livaniou, ${ }^{17}$ \\ P. Svoboda, ${ }^{18}$ R. Uhlar, ${ }^{19}$ V. Tsymbal, ${ }^{20}$ R. Kokumbaeva, ${ }^{6}$ T. Urushadze, ${ }^{9}$ \\ K. Kuratov, ${ }^{6,7,21}$ H.-C. Shin, ${ }^{22}$ Y.-W. Kang ${ }^{22}$ and B. Soonthornthum ${ }^{1}$ \\ Affiliations are listed at the end of the paper
}

Accepted 2017 October 27. Received 2017 October 25; in original form 2017 April 4

\begin{abstract}
We analysed photometric time series of the active, semidetached Algol-type system RZCas obtained in 1999-2009, in order to search for seasonal and short-term variations in the oscillation spectrum of RZCas A. The orbital period shows $\pm 1 \mathrm{~s}$ cyclic variations on time-scales of 6-9 years. We detected six low-degree p-mode oscillations with periods between 22.3 and $26.22 \mathrm{~min}$ and obtained safe mode identifications using the periodic spatial filter method. The amplitudes and frequencies of all modes vary.

We tested and confirm the hypothesis that rapid variations in the pulsation spectrum of the mass-accreting component and rapid increases in the orbital period are driven by high mass transfer and accretion outbursts caused by the cyclic magnetic activity of the Roche lobe-filling donor star. Two rapid pulsation-amplitude decays observed in 2001 and 2009 can be explained by high-mass transfer events separated by the duration of the last 9 -yr long magnetic cycle. We also tested and confirm the hypothesis of an acceleration of the outer envelope of the pulsating component. We discovered synchronous, modal $m$-dependent variations in the frequencies of three identified modes, in good agreement with results of our mode identification using the periodic spatial filter method. We suggest that $m$-dependent pulsation frequency variations are caused via the Doppler-effect by variations of the rotation speed of the outer envelope of the pulsating gainer. With this method, we obtained the first asteroseismic detection and accurate measurement of the accretion driven acceleration of the outer envelope of the massaccreting component of an Algol-type star.
\end{abstract}

Key words: binaries: eclipsing-stars: oscillations.

\section{INTRODUCTION}

Mkrtichian et al. $(2002,2004)$ were the first who called attention to an attractive, new class of pulsating stars, the so-called oEA (oscillating Eclipsing Algols) stars. The oEA stars are mass-accreting components of Algol-type systems that lie inside the instability strip and show $\delta$ Sct-like oscillations. This makes them inside the $\delta$ Sct instability strip different from classical $\delta$ Sct-type stars in welldetached eclipsing binary systems, without any history of mass transfer. Mkrtichian et al. suggested that episodes of rapid mass transfer and accretion by the pulsating gainer can potentially change its oscillation properties and modal spectra. Lists of known oEA stars and the main advantages of studying them in depth were presented in several reviews by Mkrtichian et al. (2003, 2005, 2007a). Table A1 gives an actualized list of oEA stars discovered up to now.

The active, bright Algol-type binary star RZ Cas is the prototype of this class. It consists of an A3 V primary (component A, gainer) and a K0 III Roche lobe filling secondary (component B, donor) star. The most recent determinations of stellar and system parameters of RZ Cas are listed in Table 1. The parameters were derived from combined spectroscopy and photometry by Maxteed, Hill \& Hilditch (1994), Soydugan et al. (2006), Mkrtichian et al. (2007b), Lee (2014), Wilson \& Van-Hamme (2009), from photometry by Rodríguez et al. (2004a), and from spectroscopy by Lehmann \& 
Table 1. Absolute parameters of RZ Cas taken from literature.

\begin{tabular}{lccc}
\hline Parameter & LJW14 & T09 & WV09 \\
\hline$M_{1}\left(\mathrm{M}_{\odot}\right)$ & $2.054(14)$ & $2.01(2)$ & $2.099(17)$ \\
$M_{2}\left(\mathrm{M}_{\odot}\right)$ & $0.709(6)$ & $0.69(1)$ & $0.713(7)$ \\
$R_{1}\left(\mathrm{R}_{\odot}\right)$ & $1.5593(7)$ & $1.61(1)$ & $1.440(11)$ \\
$R_{2}\left(\mathrm{R}_{\odot}\right)$ & $1.931(7)$ & $1.93(-)$ & $1.939(3)$ \\
$T_{1}(\mathrm{~K})^{a}$ & $8550(100)$ & $8907(15)$ & $8600(-)$ \\
$T_{2}(\mathrm{~K})^{a}$ & $4590(100)$ & $4797(20)$ & $4764(30)$ \\
$\log g_{1}(\mathrm{cgs})$ & $4.35(1)$ & 4.35 & - \\
$\log g_{2}(\mathrm{cgs})$ & $3.72(1)$ & 3.50 & - \\
$i\left({ }^{\circ}\right)$ & $82.53(3)$ & $82.0(3)$ & $82.09(11)$ \\
$a\left(R_{\odot}\right)$ & $6.66(1)$ & - & $6.692(16)$ \\
$L_{1}\left(\mathrm{~L}_{\odot}\right)$ & $12.2(6)$ & - & 10.46 \\
$L_{2}\left(\mathrm{~L}_{\odot}\right)$ & $1.5(1)$ & - & 1.327 \\
Distance $(\mathrm{pc})$ & $70.9(1.9)$ & - & $67.45(41)$ \\
vsini $i_{1}\left(\mathrm{~km} \mathrm{~s}^{-1}\right)$ & - & $66.0(5)$ & - \\
vsini $_{2}\left(\mathrm{~km} \mathrm{~s}^{-1}\right)$ & - & $81(2)$ & - \\
\hline
\end{tabular}

Notes. LJW - Lee J.W., private communication, 2014, T09 - Tkachenko et al. 2009, WV09 - Wilson \& Van-Hamme 2009

Mkrtichian (2004, 2008, hereafter LM04 and LM08, respectively) and Tkachenko, Lehmann \& Mkrtichian (2009).

RZ Cas was known for decades to show a short-period instability in its light curve (LC) (Olson 1982). Ohshima et al. (1998, 2001) were the first who attributed these variations to pulsation with a period of $P_{1}=22.4 \mathrm{~min}\left(f_{1}=64.19 \mathrm{~cd}^{-1}\right)$ and $\mathrm{B}$ and $\mathrm{V}$ semiamplitudes of 10.8 and $7.8 \mathrm{mmag}$, respectively.

Matter escaping the Roche lobe-filling K-giant via the Lagrange L1 point directly impacts the surface of the oscillating gainer, forms an equatorial 'belt-like' gas structure circulating into circum-binary space and the orbital plane (see fig. 2 in Mkrtichian et al. 2007a). Assuming that non-stationary accretion can influence the gainer pulsation spectrum (Mkrtichian et al. 2002), a photometric and spectroscopic monitoring was started by us since 1999, in order to search for variability in the pulsation spectrum of RZ Cas. During the multisite spectroscopic and photometric campaign in 2001 (Mkrtichian et al. 2003) the principal mode $f_{1}$ and a second oscillation mode of about $0.8 \mathrm{mmag}$ at $f_{2}=56.7 \mathrm{c} \mathrm{d}^{-1}$ was detected. It was found that in 2000-2001 the principal mode $f_{1}$ had undergone drastic changes in pulsation amplitude. In this period, the semiamplitude of $f_{1}$ decreased to the $1 \mathrm{mmag}$ level. The detection of the two oscillation frequencies, $f_{1}$ and $f_{2}$, was independently done in the 2001 in the radial velocity (RV) (LM04) and photometric (Mkrtichian et al. 2003) variations.

Based on multisite studies of RZ Cas A in 1999, Rodríguez et al. (2004a) confirmed the dominant $f_{1}$ oscillation mode with a semi-amplitude in $y$ of $6.5 \mathrm{mmag}$. Authors also suspected the existence of other low-amplitude $(0.49-0.66 \mathrm{mmag})$ oscillations at the frequencies $64.10,61.40$ and $57.58 \mathrm{c} \mathrm{d}^{-1}$. The frequency 57.58 $\mathrm{c} \mathrm{d}^{-1}$ could be interpreted as the $1 \mathrm{c} \mathrm{d}^{-1}$ alias of $56.7 \mathrm{c} \mathrm{d}^{-1}$ reported earlier by Mkrtichian et al. (2003).

LM04 focused on a precise RV study and found two dominant frequencies of $f_{2}=56.600 \mathrm{c} \mathrm{d}^{-1}$ and $f_{1}=64.189 \mathrm{c} \mathrm{d}^{-1}$ in their 2001 spectroscopic data. Both modes showed phase-dependent amplitude variations: an amplitude maximum occurred at phases of $\phi \approx 0.1$ (just after the primary minimum) and a broad minimum at $\phi=$ 0.6-0.9. In the RVs obtained from new spectroscopic observations in 2006, the spectrum was dominated by $f_{1}$ and, besides $f_{1}$ and $f_{2}$, a new frequency, $f_{3}=62.40619(36) \mathrm{c} \mathrm{d}^{-1}$, was observed (LM08). All three frequencies showed a modulation of the amplitude with an amplification around primary minimum.
Narusawa, Ozaki \& Sadakane (2006) found $\lambda$ Boo-type chemical anomalies in RZ Cas A. However, Tkachenko et al. (2009) showed that both components have abundances close to the solar values, from a careful analysis of the LM04 and LM08 spectra from 2001 and 2006. Furthermore, they detected a large cool spot on the surface of the Roche lobe-filling (RZ Cas B) component, centred at the substellar point close to the L1 point, in good agreement with theoretical prediction by Unno, Kiguchi \& Kitamura (1994) and confirmed the increase in the orbital period by $2 \mathrm{~s}$ between the years 2001 and 2006.

The current paper summarizes the main results of the photometric and spectroscopic studies of RZ Cas in the years 1999-2009, focusing on the influence of non-stationary mass-transfer on the amplitude and frequency variations of the excited modes, and proposes a possible explanation of the physical origin of these variations. The paper is organized as follows: Section 2 gives an overview of the photometric observations taken in 1997-2009. Section 3 describes the orbital period variability of RZ Cas. A detailed investigation of the pulsation spectrum of the primary component of RZ Cas and its timely variations in 1999-2009 are presented in Section 4. Section 5 is devoted to the mode identification using the periodic spatial filter method (Gamarova et al. 2003). Section 6 addresses the physical cause of the simultaneous rapid changes in the pulsation spectrum and in the orbital period. The main results of our investigation and different hypotheses are summarized and presented in Section 7. Conclusions are given in Section 8.

\section{OBSERVATIONS}

Time series of photometric observations were obtained at different observatories and telescopes using both PMT and CCD photometers. Different comparison and check stars were used, depending on the different fields of view (FOV). The 1999 observations at Abastumani Astrophysical Observatory (Georgia) were performed to provide a second site at different longitude to support the photometric observations at Sierra Nevada Observatory (Rodríguez et al. 2004a). The 2001 multisite photometric campaign was organized in the same time span as the spectroscopic observations of RZ Cas (LM04). The 2003 and 2005-2009 photometric observations were carried out to monitor the annual changes of the photometric pulsation amplitude. The aim of the 2006 spectroscopic observations was to identify the oscillation spectrum and to observe the spectroscopic effects of mass transfer.

Table A2 lists the observatories participating in the 1999-2009 observations, together with the used instruments and comparison stars. The journal of observations is given in Table A3. Table 2 lists the times of primary and secondary minima determined from our 1999-2009 photometric observations. We include in this table the eight primary minima determined by us from the unpublished 1982-1984 B and V observations by one of the authors (JEB) using the $0.48 \mathrm{~m}$ telescope of the Abastumani Astrophysical Observatory (Georgia), and two minima obtained from unpublished photoelectric observations taken by A. Pikhun and S. Udovichenko at Mayaki Station of the Astronomical Observatory of Odessa National University.

\section{ORBITAL PERIOD VARIABILITY}

The cyclic orbital period changes observed in close binary stars of RS CVn or Algol type are assumed to be caused by magnetic activity of the cool components having the spectral types later than F5 and sizeable convective envelopes. 
Table 2. New times of RZCas primary (I) and secondary (II) minima. Abbreviations of observatories are given in Table A2.

\begin{tabular}{lcccc}
\hline HJD & Error & Filter & Min I/II & Observatory \\
\hline 2445282.4255 & 0.0001 & $V$ & I & OAO \\
2445325.4559 & 0.0008 & $V$ & I & ABAO \\
2445343.3837 & 0.0007 & $V$ & I & ABAO \\
2445667.2952 & 0.0003 & $V$ & I & ABAO \\
2445949.3751 & 0.0001 & $V$ & I & ABAO \\
2445961.3274 & 0.0002 & $V$ & I & ABAO \\
2445968.4988 & 0.0003 & $V$ & I & ABAO \\
2445998.3796 & 0.0003 & $V$ & I & ABAO \\
2451446.3470 & 0.0001 & $b$ & I & ABAO \\
2451453.5198 & 0.0001 & $b$ & I & ABAO \\
2451818.0726 & 0.0002 & $V$ & I & SOAO \\
2451821.0580 & 0.0002 & $V$ & II & SOAO \\
2451825.2424 & 0.0002 & $V$ & I & SOAO \\
2451832.4130 & 0.0006 & $V$ & I & KO \\
2451832.4157 & 0.0002 & $V$ & I & OAO \\
2452190.9929 & 0.0004 & $V$ & I & SOAO \\
2452975.0867 & 0.0001 & $V$ & I & SOAO \\
2454435.0924 & 0.0003 & $V$ & II & SOAO \\
2454516.9634 & 0.0004 & $V$ & I & SOAO \\
2454673.5390 & 0.0001 & $V$ & I & POJP \\
2454697.4452 & 0.0001 & $V$ & I & POJP \\
2454829.5207 & 0.0007 & $V$ & II & POB \\
2455067.3794 & 0.0006 & $V$ & II & POB \\
2455074.5518 & 0.0006 & $V$ & II & POB \\
2455083.5120 & 0.0001 & $V$ & I & POB \\
2455083.5121 & 0.0001 & $V$ & I & POJP \\
2455101.4409 & 0.0001 & $V$ & I & POB \\
2455101.4409 & 0.0006 & $V$ & I & POJP \\
2455223.3572 & 0.0001 & $V$ & I & POB \\
2455278.3391 & 0.0001 & $V$ & I & POB \\
\hline & & & & \\
\hline
\end{tabular}

This hypothesis is widely accepted and assumes that magnetic activity cyclically changes the oblateness and hence the gravitational quadrupole moment of the star (Matese \& Whitmire 1983; Applegate \& Patterson 1987). This causes the observed alternate changes of period decrease and increase due to angular momentum exchange within convective envelopes (so called Applegate's hypothesis; Applegate 1992). The theoretical basis of this model was further developed by Lanza \& Rodono (1999) and Rüdiger et al. (2002). Lanza \& Rodono (2004) and Lanza (2006) re-examined Applegate's hypothesis and concluded that it is not adequate to explain the orbital period modulation energetically, and a new mechanism is needed. Lanza (2006), however, does not exclude the interpretation of the orbital period modulation of close binary systems in terms of a cyclic variation of the gravitational quadrupole moment of the magnetically active component in general.

The orbital period variations in RZCas are known for a long time. Hegedüs, Szatmáry \& Vinkó (1992) reported time-scales of $13420,6710,2240$ and $1830 \mathrm{~d}$ found in the RZCas $O-C$ data for the interval 1939-1991.

Here, we re-investigate the orbital period variations of RZ Cas until 2010 March, based on all published photoelectric and CCD photometric times of minima in the $O-C$ data bases collected by AAVSO and Kreiner (2004), including our 30 new minima listed in Table 2. These data spans of almost $65 \mathrm{yr}$ from JD 2429875 to 2453454. The times of minima given in the literature were transformed into heliocentric terrestial time (HEJD) using the annual corrections tabulated in the Astronomical Almanach and applying a spline interpolation. We know the individual measurement errors for

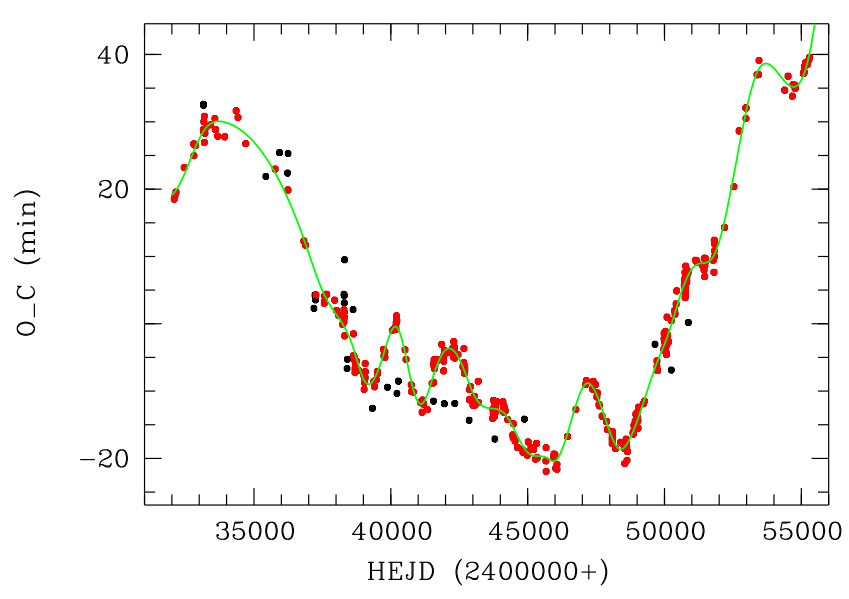

Figure 1. $O-C$ diagram of the times of minima of RZCas showing the values used for the final spline fit (green curve) in red and outliers in black colour.

only a small number of data, however. That is why, in the following, we will not weigh the data but apply a sigma clipping.

\subsection{Analysis of the $O-C$ data}

Period changes in eclipsing binaries are determined in most cases from the analysis of the $O-C$ values obtained from comparing the measured times of minima with some assumed orbital period. The $O-C$ values are implicitly given by

$T_{N}=f(N)+\epsilon_{N}$,

where $T_{N}$ is the $N$ th time of minimum, $f(N)$ is a deterministic function of the cycle number $N$ and $\epsilon_{N}$ represents the measurement error. In the linear case, it is

$f(N)=T_{0}+N P_{0}$

and the $O-C$ values are calculated using

$O-C=T-\left(T_{0}+P_{0} N\right)$

The dots in Fig. 1 show the $O-C$ values calculated with $T_{0}=2453431.646171$ and $P_{0}=1.195249 \mathrm{~d}$, the period for which the sum over all $O-C$ values from our sample is zero. Diagrams like Fig. 1 are often divided into intervals of constant period and any change of the slope with cycle number is interpreted as a period jump. However, also the presence of noise in the data (the $\epsilon_{N}$ in equation 1) can produce (feign) an amplitude variation (so-called random walk problem, see e.g. Lombard \& Koen 1993).

Instead, we want to introduce a different approach that considers a continuous change of the period with time. For that, we applied a spline fit to the $O-C$ values, iteratively removing outliers by a $4 \sigma$ clipping. The smoothness parameter of the spline fit was chosen to give the best compromise between time resolution and signal-to noise ratio. Results are shown in Fig. 1. In a next step, we took the values of the fitting spline at the observed times of minima and compared the values of neighbouring data points to derive the actual period change at the corresponding time of minimum from the local slope of the $O-C$ values with cycle number $N$ :

$\Delta P(\hat{T})=\frac{(O-C)_{k}-(O-C)_{k-1}}{N_{k}-N_{k-1}}$

with $\hat{T}=\left(T_{k}+T_{k-1}\right) / 2$. 


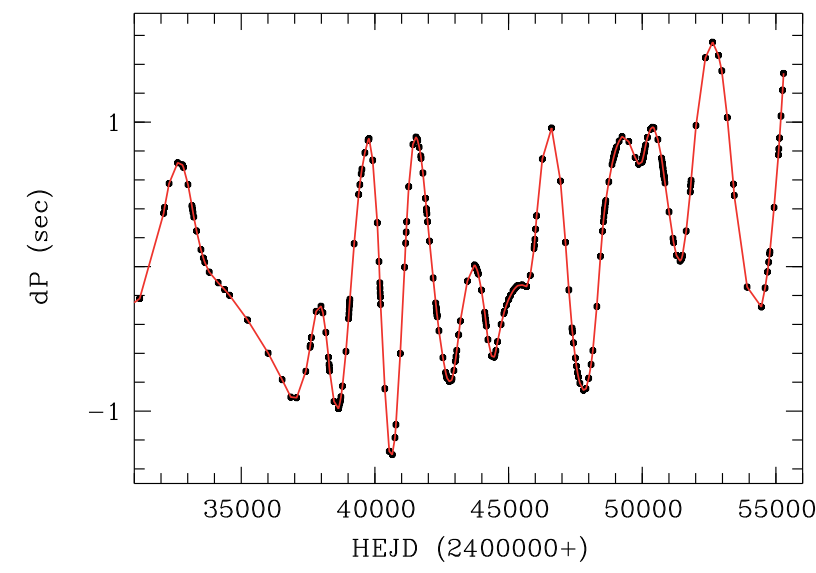

Figure 2. Deviation of the local period from the assumed period $P_{0}$. The solid curve shows a cubic spline fit.

Fig. 2 shows the results obtained from all regions where data points are sufficiently dense (we had to omit the earliest observations and also the region before JD 2437000 as it suffers from large gaps). The variation of the period can be described as a continuous one.

There is a discussion in the literature if the period changes obtained from $O-C$ diagrams are reliable or if they are feigned by the minimum time measurement errors (random walk problem). Our method determines the local period changes and should not be influenced by this random walk problem. When applying our method to artificial times of minima based on the given time sampling, we see that we need a mean measurement error of $0.005 \mathrm{~d}$ to produce a period variability of $\pm 1 \mathrm{~s}$ as obtained from the RZCas data. The mean measurement error for RZCas is 10 times smaller this value, however, and the resulting deviations from the mean period $P_{0}$ are completely randomly distributed.

The approach is comparable to measuring and comparing the difference between consecutive times of minima as was done by Lombard \& Koen (1993). The use of a spline allows us to remove outliers from the data.

Our results show that the orbital period of RZ Cas is continuously and strongly changing.

\subsection{Cyclicity in the $O-C$ data}

For the period search in the $O-C$ values we used consecutive steps of DFT analysis, including many steps of a signal prewhitening procedure consisting of fitting the data with the found frequency and removing the resulting sine-wave fit. This procedure has been repeated until all significant signals were found. We used the PERIOD04 program (Lenz \& Breger 2005) together with the detrended $O-C$ data. The results are shown in Fig. 3. Three periods (amplitudes in parentheses) $P_{1}=1782 \mathrm{~d}\left(A_{1}=0.37 \mathrm{~s}\right), P_{2}=2262 \mathrm{~d}$ $\left(A_{2}=0.34 \mathrm{~s}\right)$ and $P_{3}=3356 \mathrm{~d}\left(A_{3}=0.295 \mathrm{~s}\right)$ were identified. The amplitude spectrum of residual signal shown in the bottom panel in Fig. 3 exhibits a low amplitude, nearly equal amplitude peaks. The frequencies, periods and amplitudes of found signals are listed in Table 3.

However, the DFT analyses gives reliable results only for strictly periodic signals, which may not be the case for the orbital period variations in RZ Cas that show rather quasi-periodic variations.

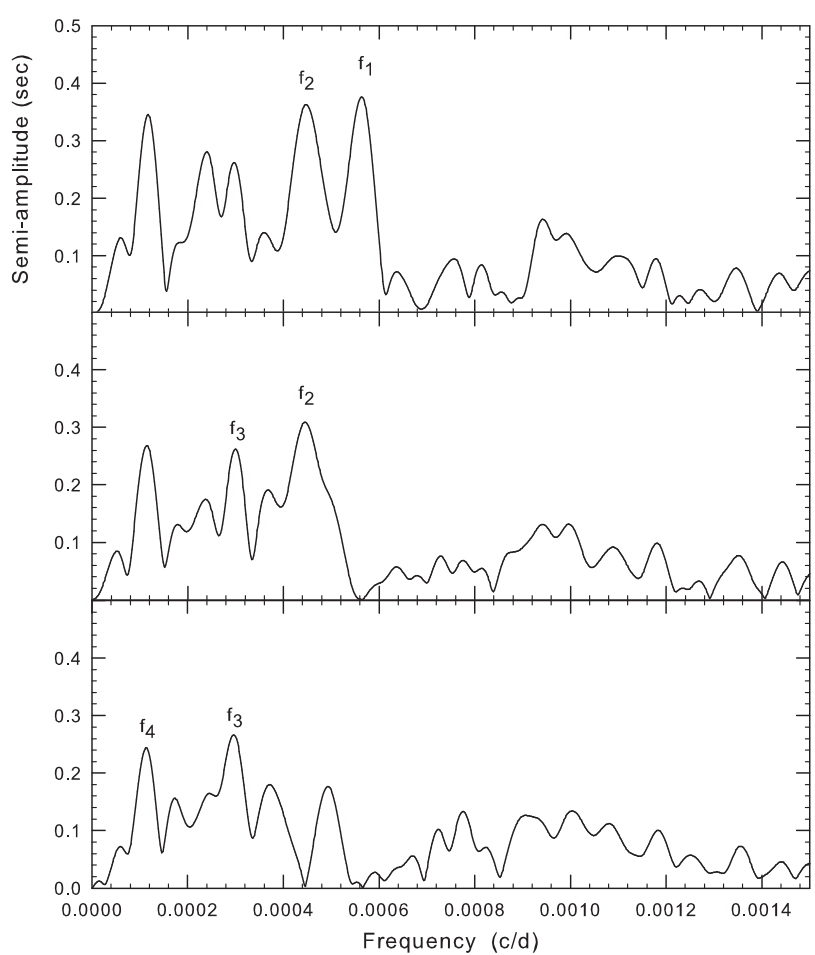

Figure 3. Amplitude spectra from consecutive steps (from top to bottom) of DFT analyses of orbital period variations. Found frequencies $f_{1}, f_{2}$ and $f_{3}$ are marked and listed in Table 4.

Table 3. Periods and semi-amplitudes of orbital period variations found in the DFT and Wavelet analyses for $C=0.003$. DFT errors in period and amplitude are least-square errors, while Wavelet analysis errors are standard deviations calculated from the skeleton map.

\begin{tabular}{cccccc}
\hline & $\begin{array}{c}f \\
\left(\mathrm{c} \mathrm{d}^{-1}\right)\end{array}$ & $\begin{array}{c}\text { Period } \\
(\mathrm{d})\end{array}$ & $\begin{array}{c}\text { Period } \\
(\mathrm{yr})\end{array}$ & $\begin{array}{c}\text { Amplitude } \\
(\mathrm{s})\end{array}$ & $\begin{array}{c}\text { Interval } \\
\text { JD }\end{array}$ \\
\hline & & & $\mathrm{DFT}$ & & \\
$f 1$ & $0.000561(1)$ & $1782(3)$ & $4.85(4)$ & $0.42(2)$ & $30000-55500$ \\
$f 2$ & $0.000442(1)$ & $2262(5)$ & $6.15(6)$ & $0.34(2)$ & - \\
$f 3$ & $0.000298(2)$ & $3356(22)$ & $9.22(3)$ & $0.31(2)$ & - \\
& & & WWA & & \\
$f 1$ & $0.000568(1)$ & $1758(5)$ & $4.81(1)$ & $0.39(1)$ & $43500-54000$ \\
$f 2$ & $0.000451(3)$ & $2215(16)$ & $6.06(5)$ & $0.49(5)$ & $30000-42500$ \\
$f 3$ & $0.000298(1)$ & $3348(20)$ & $9.16(6)$ & $0.41(4)$ & $54500-55500$
\end{tabular}

For a cross-check, we applied the Morlet Wavelet transform analysis by Grossman \& Morlet (1984) in the form given by Foster (1996)

$F(z)=\mathrm{e}^{i \omega(t-\tau)-c \omega^{2}(t-\tau)^{2}}$

Wavelets are better suitable for the analysis of quasi-periodic signals. We tested and compared two approaches. In the first case, we used rather small value for the Gaussian decay constant, $C=0.00125$, to have better frequency resolution (to the expense of the time resolution). In the second case, we used $C=0.003$ to get better time resolution (to the expense of frequency ones). The resulting weighted wavelet Z-transform (WWZ) maps (Foster 1996) and the 'skeletons' of weighted wavelet amplitude (WWA) maps of the dominant periods in the data are shown in Figs 4 and 5, respectively. 

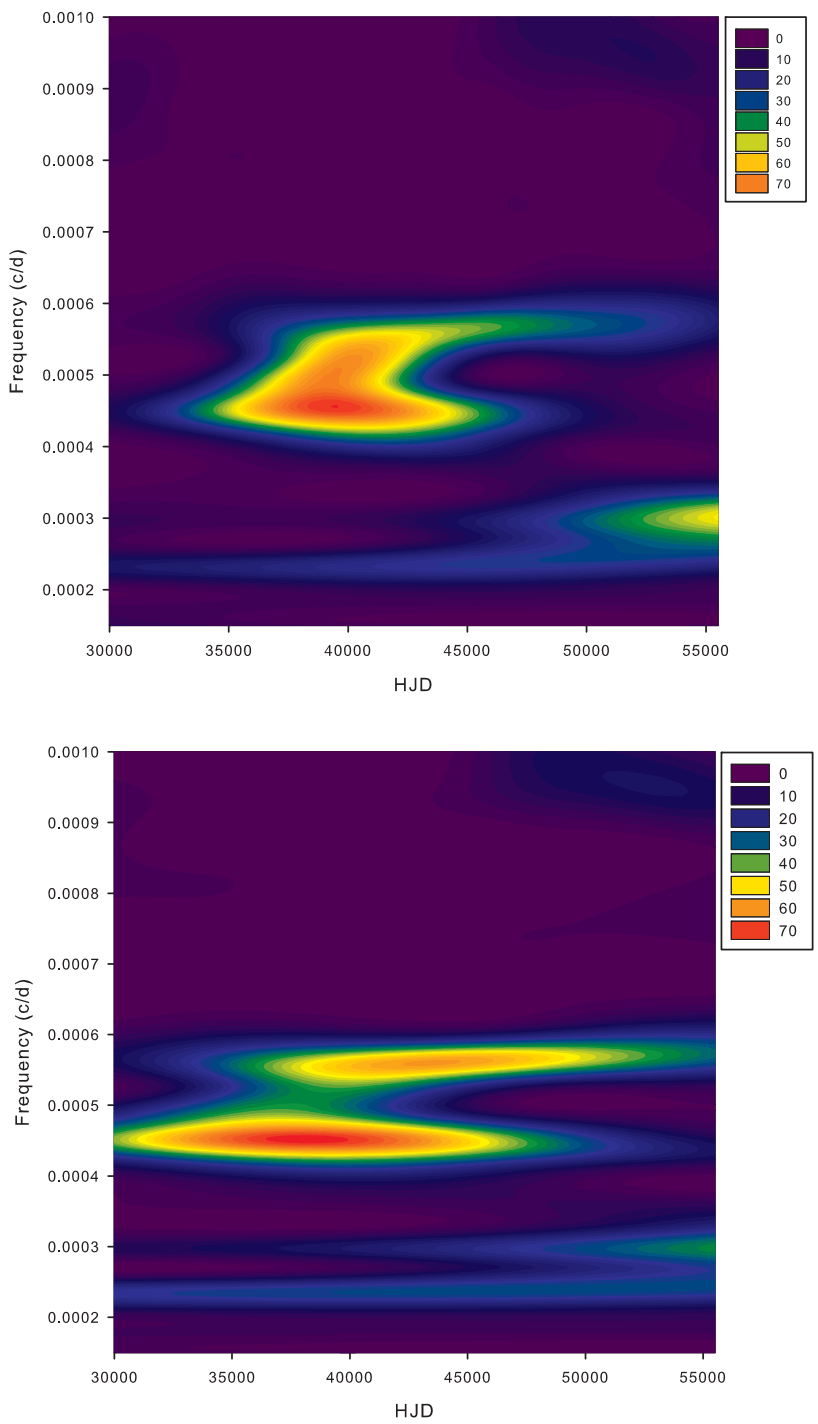

Figure 4. WWZ maps of de-trended orbital period variations calculated for two different decay constants $C=0.003$ (top) and $C=0.00125$ (bottom).

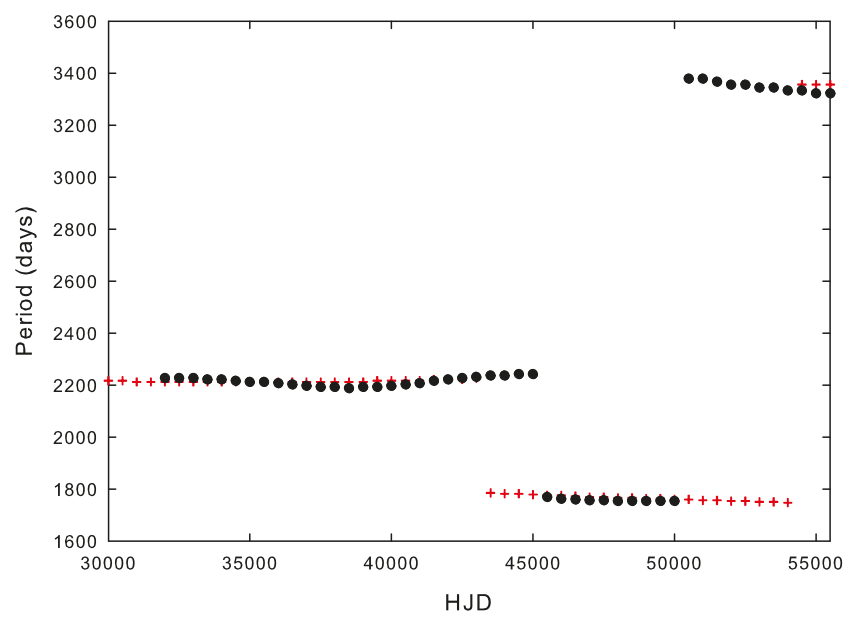

Figure 5. Skeletons of the WWA map for orbital period variations calculated with $C=0.003$ (dots) and $C=0.00125$ (crosses).
Table 3 lists results of DFT and Wavelet analyses of the $O-C$ variations for $C=0.003$. As seen, the results are in close agreement: the DFT and Wavelet analyses revealed three dominant periods of $O-C$ variation of about $4.81,6.06$ and $9.16 \mathrm{yr}$.

From the combined results of DFT and Wavelet analyses follows that the time-scale of $6.06 \mathrm{yr}(2215 \mathrm{~d})$ was dominant in JD 2430000 to 2445000 , then switched to $4.81 \mathrm{yr}$ (1758 d) variations in JD 2445000 to JD 2450000, and to $9.16 \mathrm{yr}$ (3348 d) afterwards. The detection of three distinct time-scales shows that RZCas exhibits a quasi-periodic behaviour. On the other hand, the analysis of spectroscopic data by LM08 showed an increase of the orbital period of $1.6 \mathrm{~s}$ between 2001 and 2006 over a time span of $4.33 \mathrm{yr}$. This value is nearly half of the last $9.2 \mathrm{yr}$ cycle we found from the actual $O-C$ study.

\section{PULSATION SPECTRUM ANALYSIS BASED ON THE 1999-2009 DATA}

Mkrtichian et al. (2003, 2007b) concluded that rapid changes of the pulsation amplitude in the RZ Cas system are likely induced by high-mass transfer episodes. To confirm this hypothesis, we studied the p-mode spectrum of RZCas and its variability, based on data covering $11 \mathrm{yr}$ of photometric observations. The study was based on a year-to-year data analysis.

In our search for oscillation frequencies in the LCs, we omitted all data at primary minimum (between phases 0.9 and 1.1). The observed brightness is caused by a superposition of rapid (around $22 \mathrm{~min}$ ) pulsations and slow light variations outside primary and secondary eclipses caused by the reflection effect. The slow variations were approximated by low-order polynomials or cubic splines, and removed. As in Section 3.3., we used consecutive steps of DFT analysis, each consisting of a period search followed by a signal pre-whitening procedure, to find all significant frequencies.

\subsection{The pulsation spectrum in 1999}

Two photometric runs were obtained in 1999: Strömgren $u, v, b, y$ photometry from July 29 to November 16 (JD 2451388-2451507) at SNO (Rodríguez et al. 2004a, are not listed in table 10) and six nights (from JD 2451446 to 2451525) overlapping the SNO nights were acquired by us through Strömgren $b$ filter with the $1.25 \mathrm{~m}$ telescope at the Abastumani Astrophysical Observatory (AAO) (Fig. B1). SNO data were analysed by Rodríguez et al. (2004a). The dominant oscillation mode was found at $f_{1}=64.1935(2) \mathrm{c} \mathrm{d}^{-1}$, with semi-amplitudes of $10.4,8.5,7.5$ and 6.5 mmag in $u, v, b, y$ filters, respectively. From a frequency analysis of AAO data, we found the dominant oscillation mode at $f_{1}=64.19381(8) \mathrm{c} \mathrm{d}^{-1}$ with a semiamplitude 7.02(12) mmag - in good agreement with the $b$-filter amplitude and frequency obtained by Rodríguez et al. (2004a). Removing this signal, the residual spectrum shows a 1.29 mmag peak at $63.1067 \mathrm{c} \mathrm{d}^{-1}$ which is the $1 \mathrm{c} \mathrm{d}^{-1}$ alias frequency of $64.01 \mathrm{c} \mathrm{d}^{-1}$ found by Rodríguez et al. (2004a) among other lower amplitude peaks in their data.

To study the oscillation spectrum of RZ Cas in detail, we combined the SNO and AAO $b$-filter high-quality data and did a period search of this $138 \mathrm{~d}$-spanning set. The steps of this DFT analysis are illustrated in Fig. 6. We found six statistically significant $\left(\mathrm{FAP}<510^{-5}\right)$ peaks from the successive pre-whitening. The schematic oscillation spectrum is presented in Fig. 7, the frequencies and semi-amplitudes are listed in Table 4.

The frequencies marked with a prime in Fig. 6 and Table 4 are numbered in the order of detection from successive pre-whitening. 


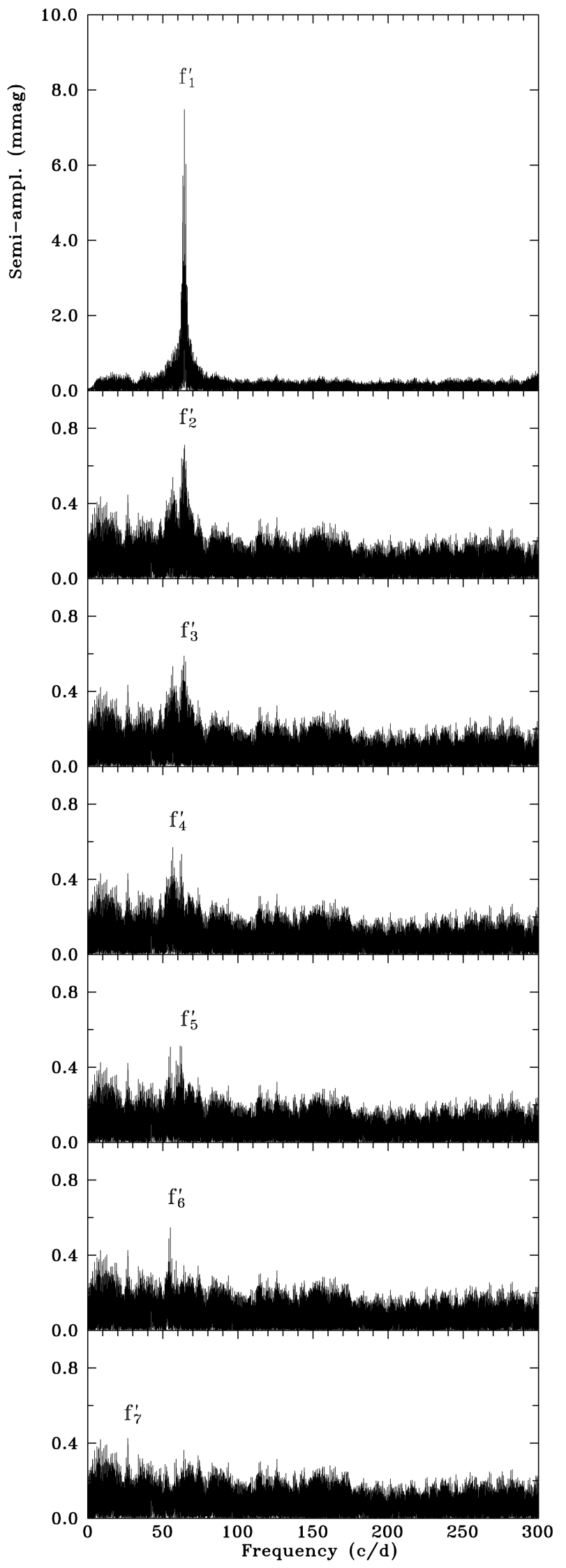

Figure 6. The DFT analysis of the combined SNO and AAO $1999 b$-filter data

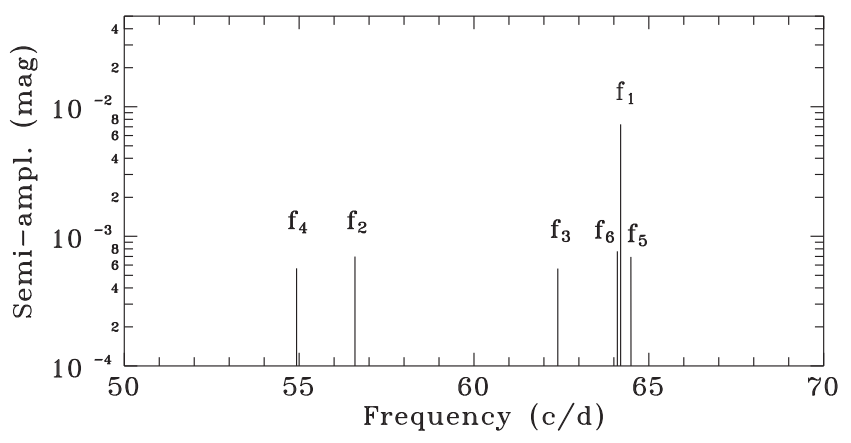

Figure 7. Schematic oscillation spectrum of RZ Cas in 1999.

Table 4. The six frequencies $f_{1}^{\prime}-f_{6}^{\prime}$ consecutively found in the combined $1999 b$ data. All frequencies present in the 1999 data, were found in later years but with different amplitudes. Hereafter, we will follow the numbering of frequencies according to their timely discovery. This assignment of $f_{1}^{\prime}-f_{6}^{\prime}$ to $f_{1}-f_{6}$ is explained in detail in the first column.

\begin{tabular}{lcc}
\hline & Frequency $\left(\mathrm{c} \mathrm{d}^{-1}\right)$ & Semi-amplitude $(\mathrm{mmag})$ \\
\hline$f_{1}=f_{1}^{\prime}$ & $64.19415(4)$ & $7.26(6)$ \\
$f_{5}=f_{2}^{\prime}$ & $64.4875(5)$ & $0.58(6)$ \\
$f_{6}=f_{3}^{\prime}$ & $64.0994(4)$ & $0.79(6)$ \\
$f_{2}=f_{4}^{\prime}$ & $56.5955(5)$ & $0.66(6)$ \\
$f_{3}=f_{5}^{\prime}$ & $62.3974(5)$ & $0.60(6)$ \\
$f_{4}=f_{6}^{\prime}$ & $54.9281(6)$ & $0.58(6)$ \\
\hline
\end{tabular}

Table 5. Frequencies found in the $2009 v$-data.

\begin{tabular}{ccc}
\hline & Frequency $\left(\mathrm{c} \mathrm{d}^{-1}\right)$ & Semi-amplitude (mmag) \\
\hline$f_{1}$ & $64.202(2)$ & $1.57(6)$ \\
$f_{4}$ & $54.931(5)$ & $0.57(6)$ \\
$f_{2}$ & $56.632(6)$ & $0.39(6)$ \\
\hline
\end{tabular}

They are re-numbered in Table 4 in the order of their historical detection. In the following, we will use this latter numbering throughout the paper.

In the night of JD 2451525, Johnson $B$-filter observations began at TSAO, overlapping with the last night of AAO $b$-filter photometry. A total of four consecutive continuous nights of photometric data (JD 2451525 to 2451529 ) was acquired. The analysis confirmed the dominant oscillation mode with $f_{1}=64.17(1) \mathrm{c} \mathrm{d}^{-1}$ and $A_{1}=8.2(4)$ mmag. This semi-amplitude is in good agreement with the $\mathrm{V}$ semi-amplitude of $8.0 \mathrm{mmag}$ expected from the SNO $\mathrm{b}$ and $\mathrm{v}$ semi-amplitudes measured in 1999 (we simply averaged the $b$ and $\mathrm{v}$ amplitudes as the maximum transparency of the $B$ filter is between the central wavelengths of these filters). The used scaling coefficients between the wavelength bands were $A(v) / A(b)=1.154$ and $A(b) / A(B)=0.938$. No other frequencies were detected within the detection threshold of $1.95 \mathrm{mmag}$. The frequencies found in the SNO and AAO data agree within the accuracy of measurement.

We conclude that in 1999, the oscillation spectrum of RZ Cas was dominated by the $f_{1}$ mode showing a large and stable amplitude. Besides this mode, five additional frequencies at sub-millimag level were found.

\subsection{The pulsation spectrum in 2000}

Five photometric runs were obtained in 2000: a short, $1.5 \mathrm{~h}$-long time series of $B$-filter photometry of RZ Cas was obtained on 2000 
Table 6. Amplitude variations of the dominant $f_{1}$ mode observed in 1997-2009. * asterisk marks $V$-filter amplitude scaled from B.

\begin{tabular}{lccl}
\hline$\overline{\mathrm{JD}}$ & $\begin{array}{c}\text { Frequency } \\
\left(\mathrm{c} \mathrm{d}^{-1}\right)\end{array}$ & \multicolumn{2}{c}{$\begin{array}{c}\text { Semi-amplitude } \\
(\mathrm{mmag})\end{array}$} \\
\hline & & $\mathrm{V}, \mathrm{y}$ & $\mathrm{B}$ \\
\hline 2450818 & $64.1972(7)$ & $7.4(3)$ & $8.6(2)$ \\
2451485 & $64.19381(8)$ & - & $7.02(12)$ \\
2451499 & $64.19415(6)$ & $6.5(1)$ & - \\
2451527 & $64.17(1)$ & - & $8.2(4)$ \\
2451751 & $65(5)$ & $5.9(4)^{*}$ & $7.3(4)$ \\
2451814 & $61(6)$ & $4.2(2)$ & - \\
2451826 & $64.1967(3)$ & $4.2(1)$ & - \\
2452190 & $64.195(1)$ & $1.02(8)$ & - \\
2452571 & $64.1965(7)$ & $2.7(3)$ & - \\
2452978 & $64.11(4)$ & $4.2(2)$ & - \\
2453766 & $64.6(3)$ & $3.2(5)^{*}$ & $4.0(5)$ \\
2454101 & $64.216(2)$ & $5.53(1)^{*}$ & $6.9(1)$ \\
2454434 & $64.12(5)$ & $3.8(7)$ & - \\
2454697 & $64.1(1.5)$ & $3.7(7)$ & - \\
2455152 & $64.202(2)$ & $1.390(8)$ & - \\
\hline
\end{tabular}

July 26 (JD 2451751) at MO, the follow-up 1.2 h-long $V$-filter photometry on September 26 (JD 2451814) was obtained at MO, in September-October 2000 at SOAO (JD 2451817 to 2451825), at TSAO (JD 2451806 to 2451835) and with a $y$ filter at SNO (JD 245180808 to 2451847 ). Table A3 gives the logs of observations, Fig. B2 shows the corresponding LCs.

The DFT analysis of MO low-resolution B-data revealed one peak at frequency at about $64.7(6) \mathrm{cd}^{-1}$, with a semi-amplitude of 7.3(4) mmag. The corresponding, scaled $V$-filter amplitude (using $k=0.806$ scaling coefficient) is of 5.9(4) mmag (see Table 6). Note that the pulsation semi-amplitude is 1.13 times lower than in the previous year.
The results of the DFT analysis of the combined TSAO and SNO data are shown in Fig. 8(a). During September-October, RZCas showed a principal oscillation mode with $f_{1}=64.1967(3) \mathrm{c} \mathrm{d}^{-1}$ and $A_{1}=4.2(1)$ mmag. The amplitude is even lower than in 2000 July.

The same pulsation semi-amplitude of 4.2(2) mmag was obtained for the $f_{1}$ mode from the short $V$-filter data on September 26 from MO. No further periodicity above the detection threshold of 2-3 mmag was found.

We conclude that the $V$-band semi-amplitude of the principal mode during the summer-autumn season in 2000 decreased from 5.87 to 4.2 mmag and was systematically smaller than in 1999 .

\subsection{The pulsation spectrum in 2001}

In 2001, a simultaneous campaign of photometric and spectroscopic observations on RZ Cas was organized to study the pulsation spectrum, the binary orbit, and the photometric and spectroscopic effects originating from mass transfer in the system in detail. First preliminary results from the photometric campaign were already reported (Mkrtichian et al. 2003), detailed results from the spectroscopic campaign were published by LM04 and LM08.

Inspection of the first LCs obtained in 2001 showed that the welldefined oscillations seen in previous years were apparently absent. Indeed, LCs from the highest quality nights presented in Fig. B3, show about $\pm 1-2$ mmag amplitude rapid brightness variations modulated on a time-scale of several hours - a possible signature of multiperiodic oscillations. The top panel of Fig. 8(b) shows the DFT amplitude spectrum of the best SNO plus MLO photometric observations outside primary eclipse. The highest peak occurs at the frequency $f_{1}=64.195(1) \mathrm{c} \mathrm{d}^{-1}$ with an amplitude $1.02(8) \mathrm{mmag}$. The second highest peak is $f_{2}(2001)=57.187(1) \mathrm{c} \mathrm{d}^{-1}$ with a semiamplitude of 0.79(8) mmag (middle panel). In the vicinity, there are other peaks of comparable amplitudes. Among this set of possible aliases, we regard $f_{2}=56.605(1) \mathrm{cd}^{-1}$ as the true frequency as it a

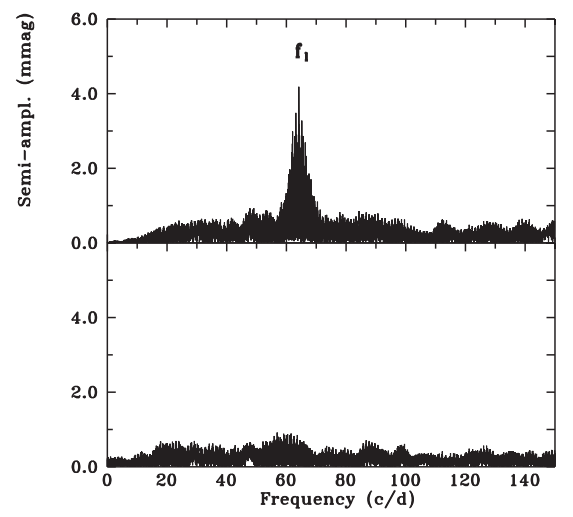

b

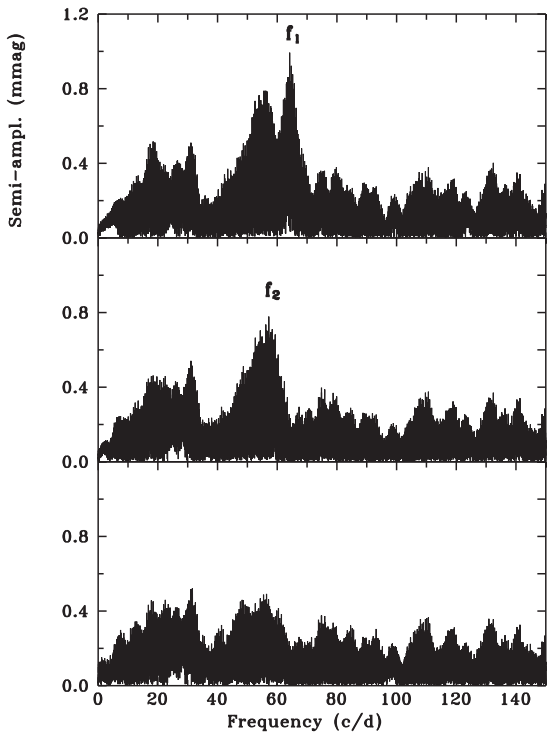

c

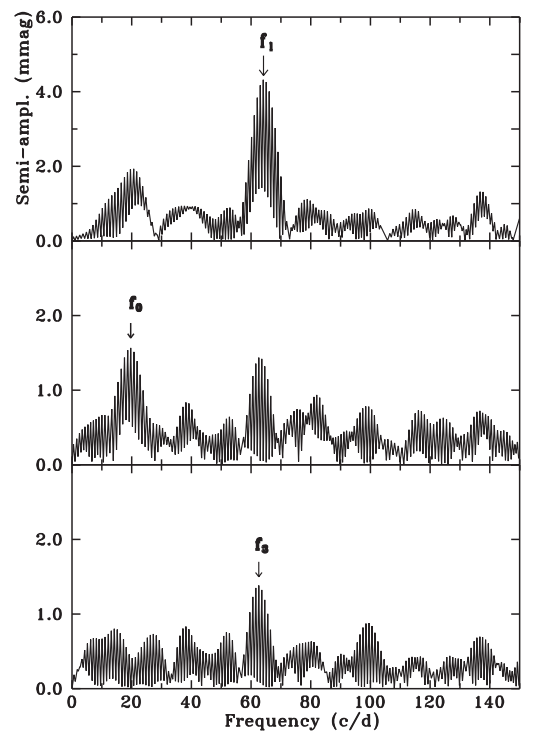

Figure 8. DFT analyses of pulsation data: (a) TSAO and SNO $V$ - and $y$-filter data from 2000. Top: original data, highest peak at $f_{1}(2000)=64.196714 \mathrm{c} \mathrm{d}{ }^{-1}$. Bottom: residual spectrum. (b) SNO and MLO $y$-filter data from 2001. Top: original data with the dominant peak at $f_{1}(2001)=64.195 \mathrm{c} \mathrm{d}^{-1}$. Middle panel: after subtracting the $f_{1}$ signal, highest peak at $f_{2}(2001)=56.195 \mathrm{c} \mathrm{d}^{-1}$. Bottom: residual spectrum. (c) The TSAO $V$-filter data from 2003. Top: original data highest peak at $f_{1}(2003)=64.195 \mathrm{c} \mathrm{d}^{-1}$. Middle panel: after subtracting the $f_{1}$ signal, highest peak at $f_{0}=19.95 \mathrm{~cd} \mathrm{~d}^{-1}$. Bottom: After pre-whitening for $f_{1}$ and $f_{0}$, highest peak at $62.58 \mathrm{c} \mathrm{d}^{-1}$. 


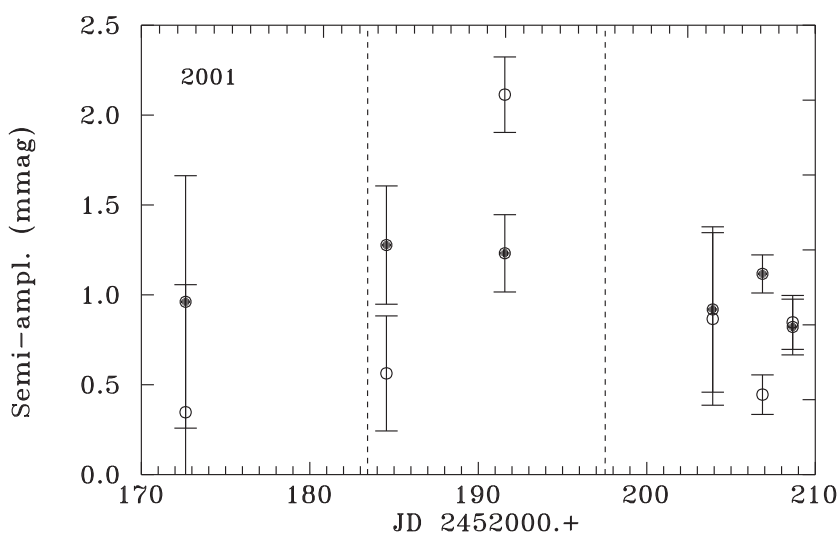

Figure 9. Variation of pulsation amplitudes of the $f_{1}$ (filled circles) and $f_{2}$ (open circles) modes observed in 2001. Two vertical dashed lines mark the time interval where the spectroscopic observations by LM04 were obtained, in the centre of this interval the inversion of mode dominancy is well visible.

agrees (within the errors) with $f_{4}$ found in 1999 and with the spectroscopic frequency found in 2001. The residual DFT amplitude spectrum after pre-whitening for the $56.605(1) \mathrm{c} \mathrm{d}^{-1}$ peak is shown in the bottom panel of Fig. 8(b).

Note that both $f_{1}$ and $f_{2}$ were independently found in parallel spectroscopic observations by LM04. Their analysis of the RZ Cas $\mathrm{RV}$ variations gives $f_{1}^{\mathrm{sp}}(2001)=64.189(6) \mathrm{c} \mathrm{d}^{-1}\left(K=99(26) \mathrm{m} \mathrm{s}^{-1}\right)$ and $f_{2}^{\mathrm{sp}}(2001)=56.600(4) \mathrm{c} \mathrm{d}^{-1}\left(K=151(27) \mathrm{m} \mathrm{s}^{-1}\right)$.

To search for variations of the pulsation amplitudes in the 2001 observations, we first analysed the entire data string which gave us the frequencies and phases of $f_{1}(2001)$ and $f_{2}$ (2001). Then, fixing the obtained frequencies and corresponding phases, we searched for the best amplitude fits to the data based on single nights. The resulting amplitudes versus the Julian date are shown in Fig. 9. The strong variability of the $f_{2}$ mode is obvious.

Remarkably, the $f_{2}$ (2001) mode shows an amplitude instability on a time-scale of about 1 week. For JD 2452191, the amplitude of the $f_{2}$ (2001) mode is larger than the amplitude of the former dominant mode $f_{1}$ (2001), with an amplitude ratio of $R=A_{f_{1}(2001)} /$ $A_{f_{2}(2001)}=0.58$, while for other dates the amplitude ratio $R$ varies between 0.97 (at JD 2452208) to 2.27 (at JD 2452184). Note that the inversion of the pulsation amplitude ratio for these two modes was also found in the RV analysis of the pulsation spectrum by LM04, with $R_{s p}=K_{f_{1}(2001)} / K_{f_{2}(2001)}=0.65$ in the period JD 24521832452197.

We conclude that these spectroscopic and photometric results provide an independent and safe confirmation of the short-term pulsation amplitude variability in 2001 .

\subsection{The pulsation spectrum in 2002, 2003 and 2006-2008}

During 2002, 2003 and 2006-2008 RZ Cas was observed at several observatories: during 15 nights from September 17 to November 29 in 2002 through $B$ filter (Soydugan et al. 2006), during three nights on 2003 December 1, 3 and 4 (JD 2452975, JD 2452977 and JD 2452978); the $V$-band LCs were obtained at TSAO, during one night on 2006 January 30 (JD 2453766); through $B$ filter at MLOAO and during five nights on 2006 November to 2007 January (JD 2454070 to 2454106) at ARIES; three consecutive nights were acquired through a $V$ filter in 2007 November at SOAO (JD 2454433-2454435) and one night (JD 2454697) of $V$-filter observations was acquired at Private Observatory in Czech Republic in 2008 .

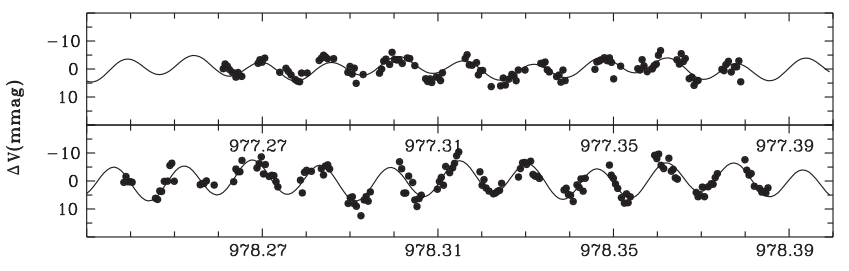

Figure 10. TSAO V pulsation LCs on JD 2452977 and JD 2452978 (2003). The solid line is the sinusoidal fit taking $f_{1}=64.107 \mathrm{c} \mathrm{d}^{-1}, f_{2}=19.679 \mathrm{~cd}$ and $f_{3}=62.55 \mathrm{~cd}^{-1}$ into account. Night-to-night amplitude changes arise from the beating of the two closest frequencies.

2002 : We re-analysed the data by Soydugan et al. (2006) after removing some trends from individual nights. In agreement with Soydugan et al., we found monoperiodic oscillations with $f_{1}=64.1965$ (7) $\mathrm{c} \mathrm{d}^{-1}$ and a $V$-band semi-amplitude of 2.7(3) mmag. No signature of the $f_{2}$ mode was detected above the noise level of $1.4 \mathrm{mmag}$.

2003 : Fig. 8(c) illustrates the steps of DFT analysis of the three nights of out-of-eclipse $V$-band light curves from TSAO. The dominant peak was found at $64.11(4) \mathrm{c} \mathrm{d}^{-1}$ with a semi-amplitude of 4.4(2) mmag (top panel of Fig. 8c). We also revealed additional signals at frequencies $19.68(4) \mathrm{c} \mathrm{d}^{-1}(A=1.5(2)$ mmag, middle panel) and $62.6(1) \mathrm{cd}^{-1}(A=1.4(2) \mathrm{mmag}$, bottom panel). We consider the $62.6(1) \mathrm{c} \mathrm{d}^{-1}$ signal as a real detection of a pulsation mode since it is close (within $\pm 2 \sigma$ ) to the $f_{5}$ mode found in the 1999 data. Its presence in the oscillation spectrum of RZCas, in addition to modes $f_{1}\left(64.2702 \mathrm{~cd}^{-1}\right)$ and $f_{4}\left(56.7610 \mathrm{~cd}^{-1}\right)$, was independently confirmed from the 2005/2006 spectroscopic RV data by LM08. The nature of the new, low-frequency $f=19.69 \mathrm{c} \mathrm{d}^{-1}$ signal, visible in only two consecutive nights, is not yet clear. Fig. 10 shows the different pulsation amplitudes occurring in two consecutive nights, caused by the beating effect of the multiperiodic oscillations.

2006 : The analysis of $B$-filter observations obtained during one night in early 2006 (JD 2453766) at MLOAO revealed the principal oscillation mode at $64.6(3) \mathrm{c} \mathrm{d}^{-1}$ with a semi-amplitude $A=4.0(5) \mathrm{mmag}$. No signals were found above the noise peak threshold of 1.5 and $1.7 \mathrm{mmag}$ in the $V$ - and $B$-filter residuals, respectively.

The analysis of the $B$-filter data from five nights in 2006 at ARIES revealed the dominant frequency at $f_{1}=64.216(2) \mathrm{c} \mathrm{d}^{-1}$ $\left(A_{1}(\mathrm{~B})=6.4(1) \mathrm{mmag}\right)$ and a second mode at 54.88(9) $\mathrm{c} \mathrm{d}^{-1}$ $(\mathrm{A}=1.6(1) \mathrm{mmag})$. Note that this frequency coincides within the measurement errors with the $f_{6}=54.92809$ mode found in the 1999 data set. The $\mathrm{V}$ semi-amplitude of the dominant mode, scaled from the $B$-filter amplitude, is $A_{1}(\mathrm{~V})=5.53(1)$ mmag and higher than found in early 2006.

2007 : Data from three consecutive nights acquired through a $V$ filter in 2007 at SOAO, show similar results to those from early 2006. The principal mode is found at $f_{1}=64.12(5) \mathrm{cd}^{-1}$ $A_{1}=3.83(7) \mathrm{mmag}$ ) and no further signal above the detection threshold of 2.6 mmag could be detected.

2008: Similar results were obtained from the analysis of one night of $V$-filter observations acquired by P. Svoboda at private observatory near Prague, Czech Republic. The dominant mode frequency was $64.1(1.5) \mathrm{c} \mathrm{d}^{-1}\left(A_{1}(\mathrm{~V})=3.7(7) \mathrm{mmag}\right)$, close to the values in 2006 and 2007.

The results from the 2002-2008 observations can be summarized as follows: the semi-amplitude of the principal pulsation mode shows changes between 2.7 and $5.5 \mathrm{mmag}$ (2007) in the $V$ filter. Modes with frequencies of 63.5 and $54.8 \mathrm{c} \mathrm{d}^{-1}$ appeared in the 2006 oscillation spectrum again, for the first time since 1999 . 


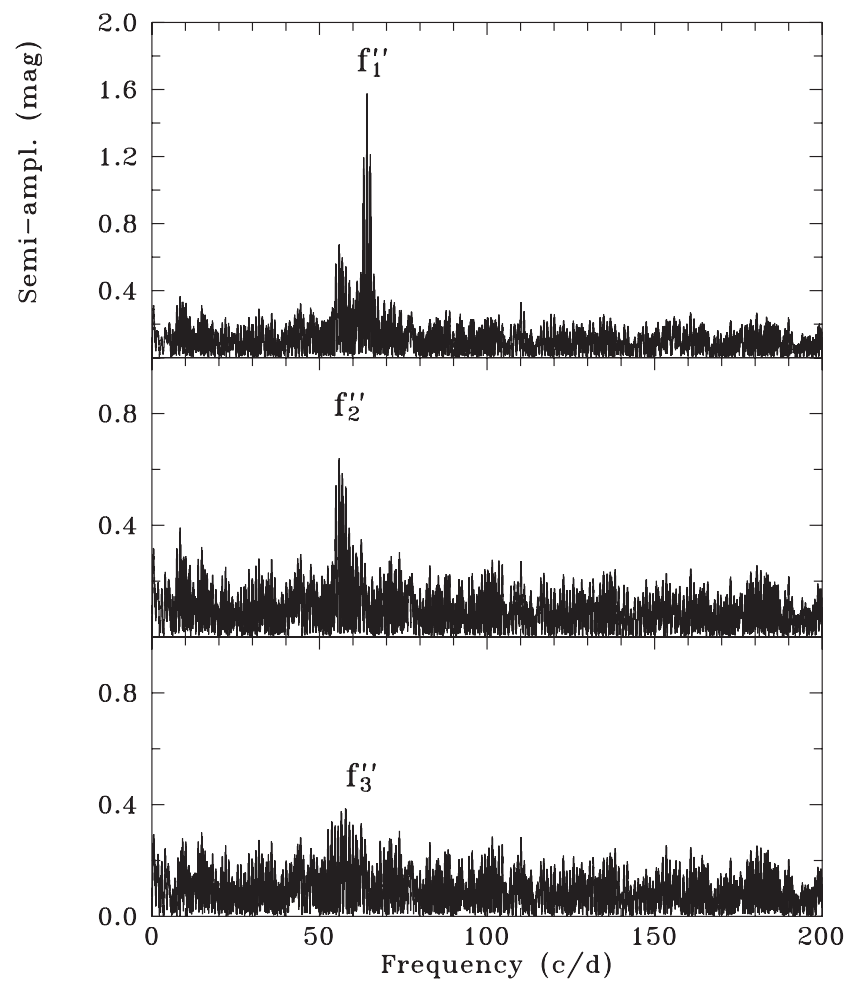

Figure 11. DFT analysis of the SNO 2009 Strömgren $v$-filter data.

\subsection{The pulsation spectrum in 2009}

Five nights of multicolour $u, v, b, y$ Strömgren photometry obtained at SNO on November 10, 11, 12, 22 and 23 (JD 2455146, JD 2455147, JD 2455148, JD 2455158 and JD 2455159) were acquired in 2009. Visual inspection of these data revealed another, drastic decrease of the amplitude of the dominant pulsation mode. The consecutive steps of the DFT analyses of the $2009 v$-filter data (Fig. 11) revealed three oscillation modes (see, Table 5) at 64.202(2) $\mathrm{cd}^{-1}$ $\left(A_{1}=1.57(6) \mathrm{mmag}\right)$ which is close to $f_{1}=64.19415(4)$ found in $1999,54.931(5) \mathrm{cd}^{-1}(A=0.57(6) \mathrm{mmag})$ close to the $f_{4}=54.9281(6) \mathrm{cd}^{-1}$ mode and 56.632(6) $\mathrm{cd}^{-1}(A=$ $0.39(6) \mathrm{mmag}$ ) close to the $f_{2}=56.5955(5) \mathrm{c} \mathrm{d}^{-1}$ mode found in the 1999 and confirmed by the 2001 and 2006 photometric and spectroscopic data. From the $y$-filter observations, we find $f_{1}(2009)=64.201(2) \mathrm{cd}^{-1}\left(A_{1}=1.35(9) \mathrm{mmag}\right)$. The mode at $f_{4}$ $=54.931(5) \mathrm{c} \mathrm{d}^{-1}$ that was first found in the 1999 data as 54.928 $\mathrm{c} \mathrm{d}^{-1}$ was also present in 2009 . We conclude, that the pattern of oscillation spectrum observed in 2009 resembles, closely the pattern of spectrum that we observed in 2001. The reason of small seasonal frequency difference in found values of $f_{1}$ and $f_{2}$ is discussed in Section 7.4.

\subsection{Orbital modulation of the pulsation amplitude}

The occurrence of mass transfer and circum-binary gas flows in the active RZ Cas system can potentially affect the apparent pulsation amplitudes when the most dense parts of the cool gas stream and the circumprimary accretion 'belt' are projected on the surface of the non-radially pulsating gainer, attenuating its observed flux (Mkrtichian et al. 2005). This effect was also reported from spectroscopic observations (LM04, LM08).

Orbital variation of pulsation amplitude is expected to result from a combination of two effect: of gas-stream/envelope attenuation

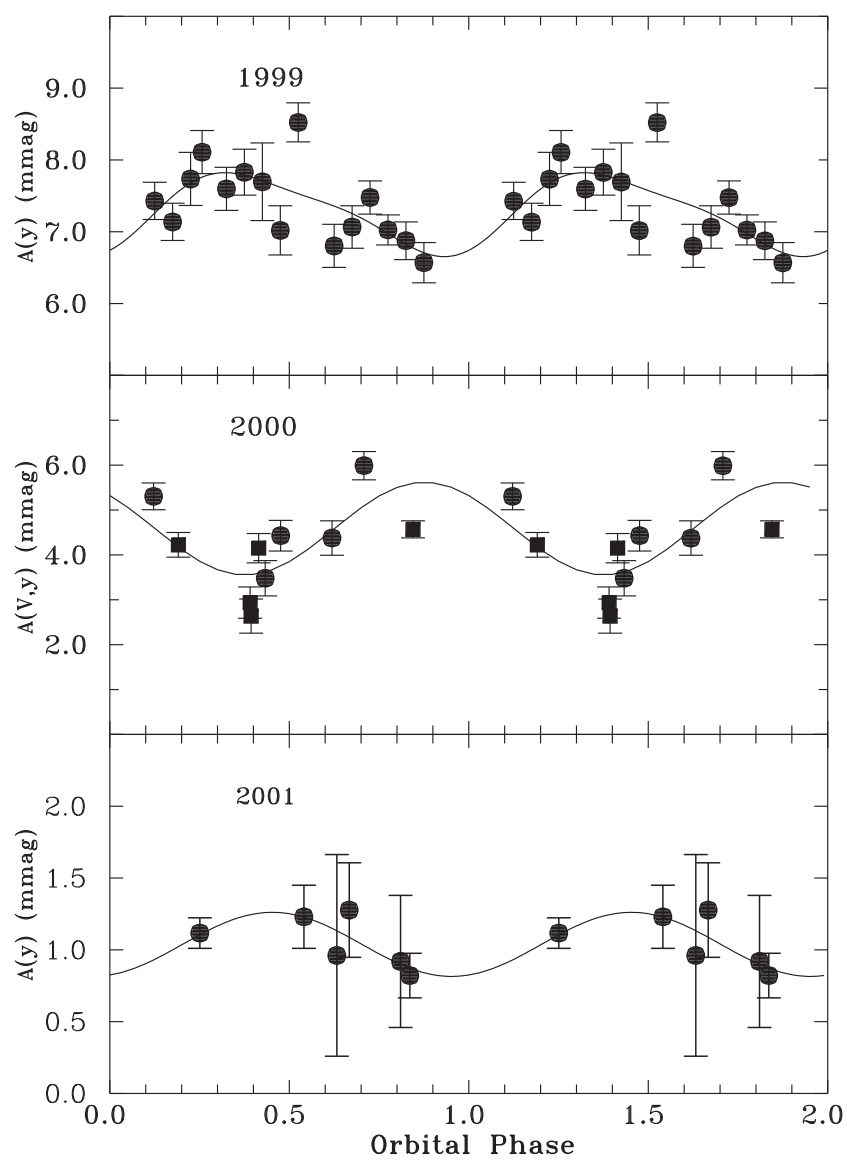

Figure 12. Orbital modulation of the pulsation amplitude of the $f_{1}=64.1935 \mathrm{c} \mathrm{d}^{-1}$ mode. Top panel: $1999 y$-filter (dots) data. Middle panel: $2000 y$-filter (dots) and $V$-filter (filled squares) data. Bottom panel: $2001 y$-filter (dots) data. The solid line in the top panel is a sine-wave plus first harmonic fit to the orbital data, while in the middle and bottom panels only sine-waves were used.

effect and the so-called periodic spatial filter effect (Mkrtichian et al. 2002; Gamarova et al. 2003), which will be discussed in Section 5.2. To investigate the dependence of the amplitude of the dominant mode $f_{1}$ on orbital phase, we folded the photometric data of every observing season against the orbital period and grouped them into orbital phase bins, where in-eclipse phases were excluded. The bin width was set in a way to obtain a best compromise between the resolution in orbital phase and a sufficient number of data points per bin. Such compromise could only be achieved for the data taken in 1999, 2000 and 2001, however, and we could not use the data from later years. Then, fixing the $f_{1}$ frequency and phase found for all seasons of observations (see Table 6), a sinusoidal least squares fit was applied to each light-curve data bin in order to calculate the optimum amplitude.

The results for the 1999, 2000 and 2001 observing seasons are illustrated in Fig. 12. As can be seen, the pulsation amplitude in the $y$-bandpass varies with orbital phase, and its shape changed from season to season. In 1999, the amplitude of the modulation has a semi-amplitude corresponding to 15 percent of the pulsation amplitude, with the maximum and minimum at orbital phases $\phi \approx 0.25-0.35$ and $\phi \approx 0.9$, respectively. In 2000 , the pulsation amplitudes are on average lower but strongly scattered, minimum and maximum of the amplitude occur at $\phi \approx 0.4$ and $\phi \approx 0.9$, respectively. In 2001, the pulsation amplitude is on average smallest over 
the entire 1997-2009 interval, with the minimum and the maximum of the amplitude occurring at $\phi \approx 0.9$ and $\phi \approx 0.45$, respectively. Orbital variation of the pulsation amplitude is present in all analysed seasons, while the shape of these variations for different seasons are phase-shifted. The seasonal amplitude modulation can be explained by variable extinction and strong variations of the mass-transfer and in density and distribution of the circum-binary environment.

\section{MODE IDENTIFICATION}

\subsection{Mode visibility in eclipsing binaries}

There is some bias in the visibility of different non-radial modes when observed in EBs using photometry and RVs. The reason is that EBs are observationally biased by a preselection of inclination angles because the orbital planes of these binary systems have orbital inclinations equal to or close to 90 degrees. The pulsating components, if their pulsation axes of symmetry are perpendicular to orbital planes (usually assumed for close binary stars, but not proved for Algols), are viewed nearly equator-on. In this case, all modes that show odd combinations of $l+|\mathrm{m}|$ are strongly damped in the disc-integrated light. The $l+|\mathrm{m}|=$ even modes, on the other side, are less affected by the cancellation effect and may be well visible (Mkrtichian et al. 2003, 2005). Thus, for a mode of degree $l, N=2 l+1$ rotationally split modes are available, but among them $N_{\text {odd }}=l$ modes are damped and possibly invisible, and only $N_{\text {even }}=l+1$ modes might be detected in the light or RV variations.

We illustrated this effect for the radial velocity amplitudes. Calculations were made with the SPOTNRP code (Mkrtichian 1992, 1993) that utilizes the formalism described by Dziembowski (1977). We assumed the rotation axis to be perpendicular to the orbital plane and to be the symmetry axis of the non-radial pulsation (NRP) modes. We assumed only the radial component of the NRP displacement and neglected the horizontal components for the intermediate overtone acoustic modes excited in RZCas. The maximal surface pulsation amplitude of modes was assumed for these simulations as $A_{\max }(l, m)=4 \mathrm{~km} \mathrm{~s}^{-1}$.

The stellar surface was divided into $120 \times 90$ surface areas over the longitude and latitude, respectively. The NRP velocity and the local stellar line profiles were calculated for each surface area and integrated over the visible stellar surface. The limb darkening coefficient was assumed $u=0.6$ and the Voight local profile was used. The centroids of the spectral lines were used to calculate the RV shifts. 20 phase intervals per pulsation cycle were used to calculate the pulsation RV curve and the pulsation amplitudes. Fig. 13 shows the ratio $A(l, m) / A_{\max }(l, m)$ of the observed discintegrated RV amplitude $A(l, m)$ to the maximal surface pulsation amplitude of mode $A_{\max }(l, m)$ calculated for $l=1$ to $l=3$ modes and for different orbital inclinations. It can be seen that for inclinations close to $90^{\circ}$ preferably modes with $l+|m|=$ even, namely $l,|m|=(1,1),(2,2),(2,0),(3,3)$ and $(3,1)$ should be observed. These modes, if excited to the detection level, should also dominate the oscillation spectrum of RZCas.

For the inclination of the orbit of RZCas, $i=82.5$, and higher modal amplitudes, there is a non-zero probability for the visibility of some $(l+|m|)=$ odd modes. In reality, the intrinsic amplitudes of the excited modes are not equal, $l+|m|=$ odd modes could have essentially larger amplitudes than even ones and additional modes with $(l,|m|)=(1,0),(2,1),(3,2)$ might become visible. Thus, using only the mode visibility criteria for mode identification is insufficient and needs to be cross-checked with other criteria, in

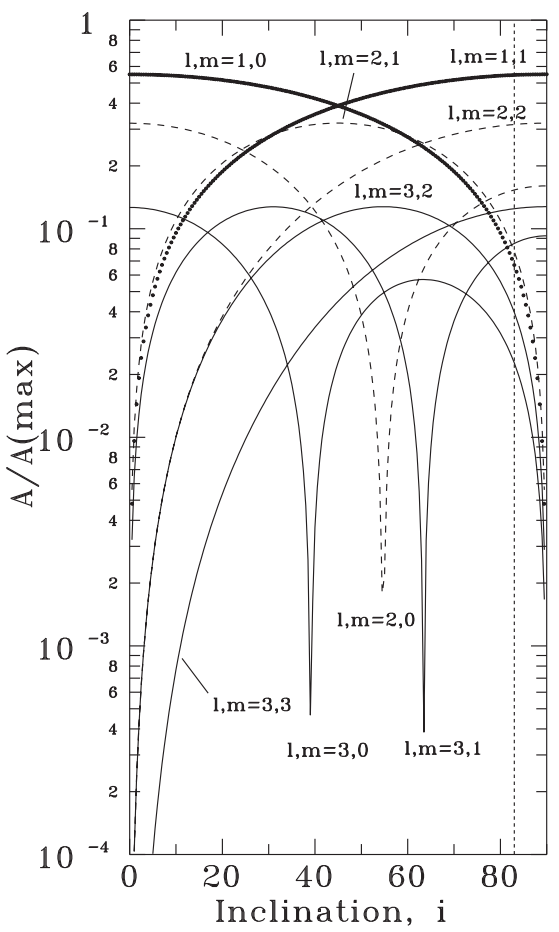

Figure 13. Mode visibility of low-degree modes for different inclinations of the rotation/pulsation axes to the line of sight. The dashed line marks the inclination of the RZ Cas orbit of 82.5 .

particular spectroscopic line-profile variations and eclipse modeidentification using the periodic spatial filter method.

\subsection{Mode identification using the periodic spatial filter method}

The eclipse mode identification method for EBs with pulsating components was suggested by Nather \& Robinson (1974). The method is based on the fact that during an eclipse of the pulsating component the occulting star acts as a screen with a timely variable shape. During an eclipse, depending on the $(l, m)$ spatial structure of modes, different variations of the pulsation amplitudes and phases are observed. The comparison of modelled and observed amplitude and phase patterns provides the correct mode identification.

The first attempt to apply the photometric eclipse mode identification on an oEA star (RZCas) and a brief description of the periodic spatial filter (PSF hereafter) method was published by Gamarova et al. (2003). Later on, this method was applied to the oEA stars AB Cas (Rodríguez et al. 2004b) and RZ Cas (Rodríguez et al. 2004a). Here, we re-analyse in detail the results of the previous preliminary mode identification for the RZ Cas system reported by Gamarova et al. (2003) and Rodríguez et al. (2004a). Gamarova et al. (2003) and Rodríguez et al. (2004a) reported that the application of the PSF method to the ascending and descending branches of the primary minima of the LC gives different preliminary identifications, namely $(l, m)=(2, \pm 1)$ and $(l, m)=(2, \pm 2)$, respectively.

Here, we want to use more conservative approach and exclude the descending branches (orbital phase intervals 0.9-1.0) from the analysis. We justify this restriction by the fact that during phases of descending branches of primary minima the surface of the primary component is screened by the optically dense gas stream and by the turbulent gas cloud formed in the zone of impact of the supersonic gas stream on the photosphere of the pulsating gainer. This can introduce an additional, unaccounted 'parasite' screening (spatial 

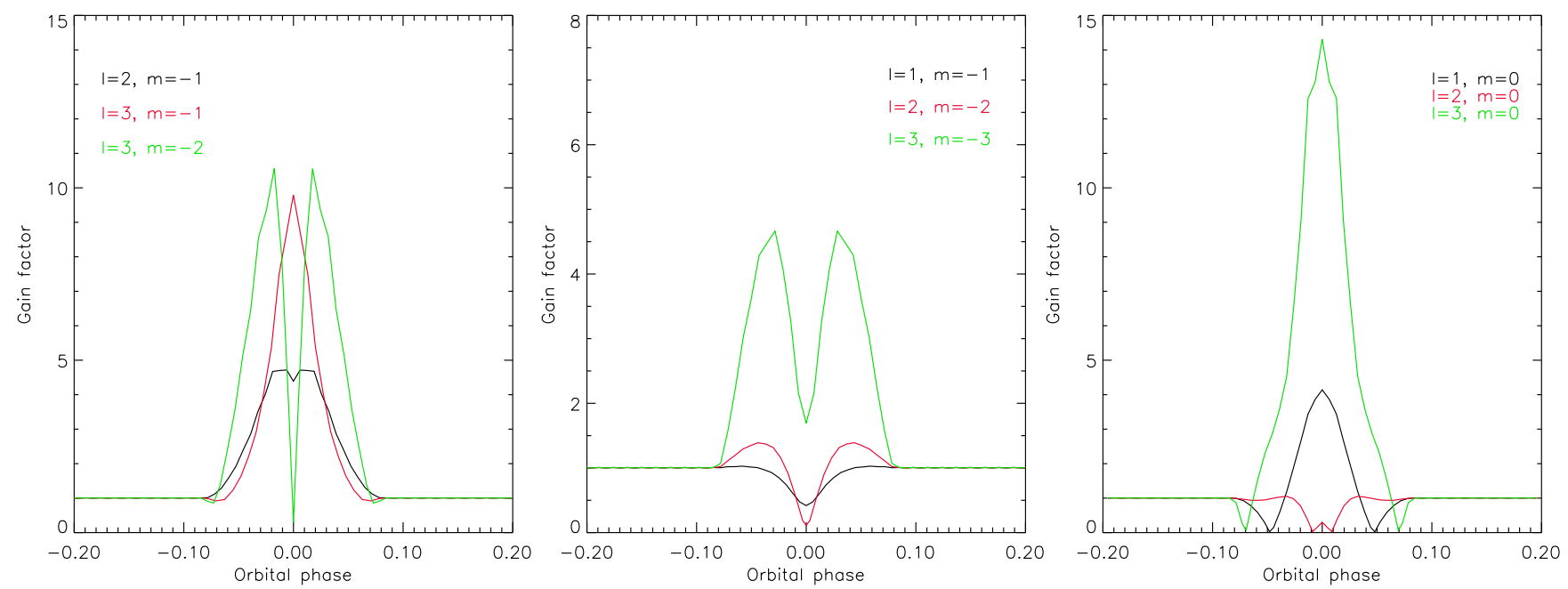

Figure 14. Modelling of the photometric gain factor (ratio of apparent in-eclipse to out-of-eclipse pulsation amplitude) for $l=0-3$ tesseral prograde (left), sectoral prograde (middle) and zonal (right) modes.

filter) effect that cannot be modelled without detailed knowledge of the geometry, density and other physical parameters of the gas stream and the turbulent cloud which is out of the scope of present paper.

On the other hand, the gas stream and the turbulent gas-stream impact zone on the stellar atmosphere are on the 'dark side' of the gainer during the phases of ascending branch of primary minima and the corresponding screening effect is minimal. The preliminary 3-D hydrodynamic simulations for the RZ Cas system (Mkrtichian et al. 2007a) show the lowest column density of the gas projected on the surface of the pulsating component just after mid-eclipse, thus perturbations by circumprimary gas at these phases can be neglected.

The main adjustable parameters in our modelling were the $(l, m)$ numbers and the pulsation amplitude of the mode. We assumed the pulsation axis aligned with the rotation axis which was assumed to be perpendicular to the orbital plane. Here, we repeat briefly the formalism of calculations used by Gamarova et al. (2003). We assume that both components have a spherical shape.

Note that the projection of distorted Roche lobe filling secondary component on the primary is close to circular shape and the difference between PSF function for spherical and non-spherical components is expected to be of the second order of magnitude for low-degree modes and has no effect in terms of amplitude and time-scales of variations. This is a reason why we ignored the nonsphericity in this paper.

The relative dimensions of primary and secondary components, orbital separation, inclination of orbit and the effective temperatures of the components were taken from the binary model (see Table 1).

The stellar surfaces of primary and secondary components were divided into $120 \times 120$ surface areas. Blackbody radiation was assumed and the reflection effect was ignored as we think it has insignificant effect around the primary eclipse phases. The rotation of primary (A) and secondary (B) components was assumed to be synchronized with the orbital period and the calculations were performed for the observed pulsation frequency $f_{1}=64.19415 \mathrm{c} \mathrm{d}^{-1}$. For each mode, the first-order frequency correction $\delta f=-m f_{\text {rot }}$ with $f_{\text {orb }}=f_{\text {rot }}=0.8366 \mathrm{c} \mathrm{d}^{-1}$ was applied to get the true value of the modal frequency in the frame of the rotating star.

To calculate the pulsational brightness variations for non-radial modes, we utilized the formalism described by Dziembowski
(1977). We assumed that in linear adiabatic approximation the brightness $I(\theta, \varphi, t)$ of a surface element with polar coordinates $\theta$ and $\varphi$ is defined as

$I(\theta, \varphi, t)=A_{0} Y_{l, m}(\theta, \varphi) \mathrm{e}^{-l \omega t}=C P_{l}^{m}(\cos \theta) \mathrm{e}^{l(m \varphi-\omega t)}$,

where $A_{0}$ is the amplitude, $Y_{l, m}(\theta, \varphi)$ spherical harmonics and $P_{l}^{m}(\cos \theta)$ associated Legendre polynomials. $C$ is a normalization constant and $\omega=2 \pi f$ is the angular frequency of a mode of spatial structure $l, m, n,(|m| \leq l)$.

At time $t$, the apparent monochromatic flux integrated over the visible part of the surface is

$I(t)=\int_{S(t)} I(\theta, \varphi, t)(1-\mu+\mu \cos \xi) \cos \xi \sin \theta \mathrm{d} \theta \mathrm{d} \varphi$,

where $\xi$ is the angle from the subobserver's point, $\mu$ is the limb darkening coefficient and the integral is evaluated over the visible (non-occulted) surface $S$ of the star, which is a function of time. The monochromatic luminosity was convolved with the transmission of the Stroemgren filter and integrated over the corresponding passband to get passband LCs. The binary LC was subtracted to get pure pulsation light variations. The pulsation amplitude of the modelled LCs was calculated as half of the magnitude difference between two consecutive positive and negative extrema of the LC. The pulsation phase shift was calculated as the difference between the observed time of maximum brightness and that predicted by the pulsation ephemeris, divided by the observed pulsation period. Due to difficulties in the determination of the observed pulsation phase shifts that are affected by uncertainties in the LC determination, we restricted our identification to the comparison of observed and modelled apparent amplitudes.

The modelling of expected apparent pulsation amplitudes $A^{\text {ecl }}(\phi)$ during the eclipse phases $\phi$ was performed for individual NRP modes of degree $l \leq 3$. As a measure for the resulting PSF amplitude amplification or reduction, we introduce the gain factor $g(\phi)=A^{\text {ecl }}(\phi) / A^{\text {out }}$. Results are shown in Fig. 14 .

Fig. 15 shows two observed $y$-filter pulsation curves obtained from the ascending branches (bottom panels) of eclipse LCs after subtracting the binary model and the comparative example for the descending branch (top panel). As seen, the photometric LC amplification for the $f_{1}=64.19415 \mathrm{c} \mathrm{d}^{-1}$ mode reaches a factor of about 3.3 at the primary minimum. 

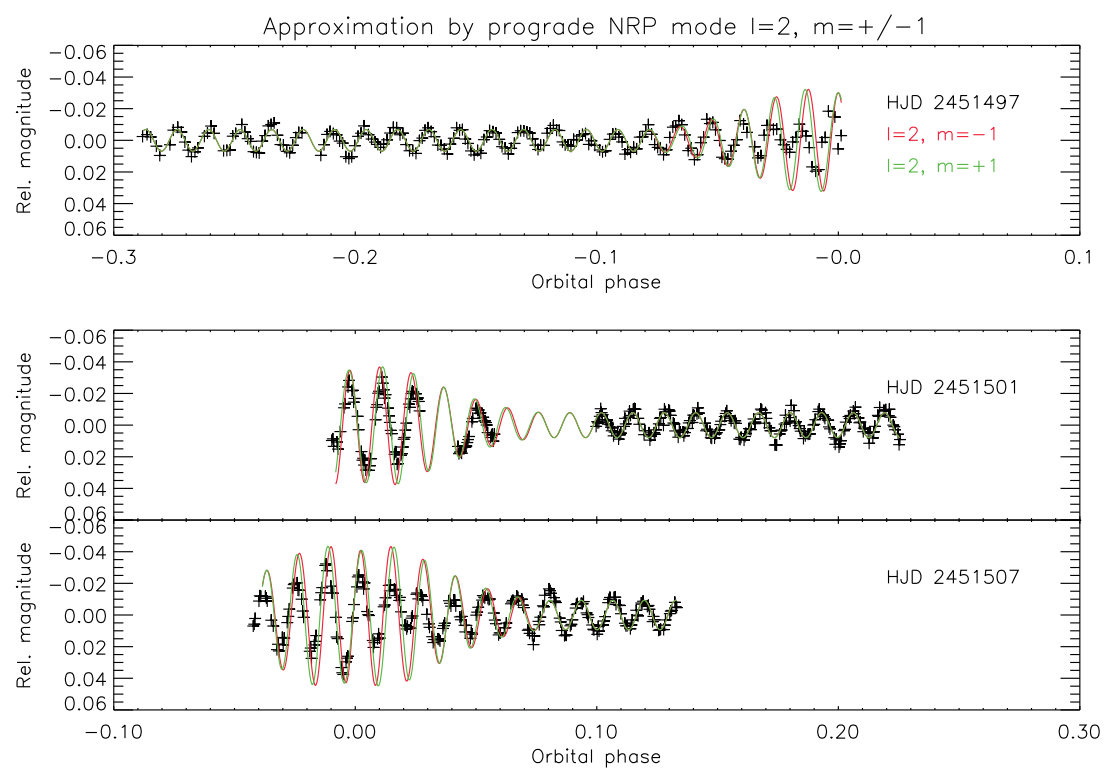

Figure 15. Residuals of the observed Strömgren $y$ LCs after subtracting the orbit for one descending (JD 2451497) and two ascending (JD 2451501 and JD 2451507) branches of primary minima of RZCas overplotted with modelled curves for $f_{1}=64.1935 \mathrm{~cd}^{-1}$ assuming a prograde tesseral mode $(l=2$, $m=-1)$ and a retrograde tesseral mode $(l=2, m=1)$.

In the following, upper indices ' $p$ ' and 's' will mark the photometric and the spectroscopic values, respectively. A comparison with Fig. 14 shows that sectorial modes cannot be responsible for the observed variations as all of them show a decrease of amplitude in the centre of primary minima. On the other hand, zonal $l=1, m=0$ modes exhibit maximum amplitude amplification in the centre of primary minima but the amplitude drops below the out-of-eclipse values shortly before and after the centre of primary minimum, which is not observed. The $l=2, m=0$ mode exhibits no amplification at all, and the amplitude decreases in the centre of minima. Thus, the zonal $(l, m)=(1,0)$ and $(2,0)$ modes can also be excluded for the identification of the $f_{1}$ mode. Among the remaining modes, the tesseral $l=2, m= \pm 1$ modes (note the very shallow and short drop of amplification in the centre is practically not detectable) show a 4.8 times rise of the amplitude. The tesseral $l=3, m= \pm 1$ modes, on the other hand, show a 10 times rise of amplitude in the centre of the minimum and do not fit the amplitude amplification criteria. Thus, the tesseral $l=2, m= \pm 1$ modes having calculated photometric $g_{\max }^{p}(2,1)=4.8$ versus observed $g_{\max }\left(f_{1}\right)=3.3$ are the most likely identification for the $f_{1}$ mode.

The solid lines in Fig. 15 show the two best fitting solutions obtained from PSF modelling. Our identification gives the best fit to the LCs observed during the ascending branches but fails to fit the descending branch. Possible reasons have been mentioned earlier in this section. The maximum photometric surface amplitude of $f_{1} l=2, m= \pm 1$ mode was found to be $A_{\max }(2, \pm 1)=0.2 \mathrm{mag}$. To save space, we do not show results of LC modelling for other modal angular and azimuthal numbers $(l, m)$, as they are largely inconsistent with observed pulsation light variations.

For completeness of identification, we use the PSF effect with respect to the RZ Cas RV amplitudes found by LM08. Fig. 6 in LM08 shows the RV-amplitude amplification during eclipse for the $f_{1}^{s}=64.27023 \mathrm{~cd}^{-1}, f_{2}^{s}=56.7610 \mathrm{~cd}^{-1}$ and $f_{3}^{s}=62.41062$ $\mathrm{c} \mathrm{d}^{-1}$ modes. Note, that LM08, in order to enhance the signal-tonoise $(\mathrm{S} / \mathrm{N})$ ratio determined the pulsation amplitude for overlapping bins of width $\Delta \phi=0.2$ in orbital phase covering the full orbital period in steps of 0.005 . This binning might smooth the rapid PSF variations and lead to an underestimation of the real amplification factor. We also refer the reader to the fact that the PSF-effect that we analyse is based on 2006 year observations, for which the circumbinary gas has essentially lower density compared to 2001 (LM08, LM04) and minor effect on the PSF shape.

As seen from fig. 6 and table 4 by LM08, the pulsation semiamplitude of the $f_{1}^{s}$ mode increased from 203 to $770 \mathrm{~ms}^{-1}$ close to the centre of eclipse, $f_{2}^{s}$ increased from 94 to $725 \mathrm{~ms}^{-1}$ with a double-peaked shape centred around mid-eclipse whereas $f_{3}^{s}$ rises in amplitude from 145 to $315 \mathrm{~ms}^{-1}$. The mid-eclipse spectroscopic RV amplitude amplifications (gain factors) are $g_{1}^{s}(l, m)=3.79$, $g_{2}^{s}(l, m)=7.71$, and $g_{3}^{s}(l, m)=2.17$ for modes $f_{1}^{s}$ to $f_{3}^{s}$, respectively. We see that $g_{1}^{s}(l, m) \approx g_{1}^{p}(l, m)$ and also the shapes of the spectroscopic and photometric amplitude variations are very similar. This is an independent support for our $l=2, m=-1$ mode identification. For the $f_{2}$ mode we have no photometric PSF amplification curve, only a spectroscopic one. The combination of the observed double-peaked shape and the derived gain factor of 7.71 (close to $\left.g_{2}^{s}(l, m)=10.5\right)$ led us to identify $f_{2}^{s}$ as an $l=3, m=-2$ mode. Note that due to the low phase resolution the dip observed at mid-eclipse ( $\delta \phi= \pm 0.016)$ is not well resolved and thus its depth and the corresponding amplitude are underestimated.

For the third spectroscopic mode, $f_{3}^{s}=62.4062 \mathrm{~cd}^{-1}$, the comparison of the shape and amplitude of the PSF amplification effect, $g_{3}^{s}(l, m)=2.2$, with Fig. 18 shows that $l=1, m=0$ with $g_{3}^{s}(l, m)=4.0$ is the most likely identification. This identification is also confirmed by the results of the frequency variation analysis as discussed in Section 7.2.

The spectroscopic and photometric observed-to-expected gain factor ratios (or amplitude underestimation coefficients) are $R_{1}^{s}=$ $0.82, R_{1}^{p}=0.69, R_{2}^{s}=0.77$ and $R_{3}^{s}=0.54$ for the $f_{1}-f_{3}$ modes, respectively. Our calculations of the RV mode visibility ratios for the inclination angle of RZ Cas of $i=82.5$ (see Fig. 13) give $A^{2,1} / A_{\max }^{2,1}$ $=0.083, A^{3,2} / A_{\max }^{3,2}=0.042$ and $A^{1,0} / A_{\max }^{1,0}=0.072$. Using these ratios and the out-of-eclipse modal RV amplitudes from table 4 by LM08, we can calculate the maximum surface RV amplitudes of the $f_{1}^{s}, f_{2}^{s}$ and $f_{3}^{s}$ modes for the 2006 season (note that the $f_{3}^{s}$ mode 
was not detected in 2001) as $A_{\max }^{2,1}=2446 \mathrm{~ms}^{-1}$ for $f_{1}^{s}, A_{\max }^{3,2}=$ $2213 \mathrm{~ms}^{-1}$ for $f_{2}^{s}$ and $A_{\max }^{1,0}=2019 \mathrm{~ms}^{-1}$ for $f_{3}^{s}$. All amplitudes are typical for classical $\delta$ Scuti stars.

Summarizingthe results of the PSF modelling and independent comparison of the photometric and RV amplification effects, we conclude that the identification of modal angular and azimuthal numbers of the observed $f_{1}, f_{2}$ and $f_{3}$ modes are most likely $(l, m)=(2,-1),(3,-2)$ and $(1,0)$ respectively. Note that all these modes have $(l+|\mathrm{m}|)=$ odd sums and thus reduced disc-integrated amplitudes.

\section{SIMULTANEOUS RAPID CHANGES IN ORBITAL PERIOD AND MODAL PATTERN}

\subsection{Amplitude changes}

We clearly detected six oscillation modes in the 1999 photometric data. Since we are confronted with variable thresholds for the different observing seasons during the 2000-2009 monitoring period some of the previously observed modes could not be found.

The comparison of the pulsation patterns observed in the period from 2000 to 2009 (including the spectroscopic results by LM04 and LM08) with the oscillation spectrum from 1999 shows that all significant frequencies coincide with the frequencies found in 1999 , except for some cases that we will discuss below.

Fig. 16 shows the timely evolution of the amplitude of the principal oscillation mode $f_{1}$ in the form of folded LCs in the filters $V$ and $y$ as observed from 1999 to 2009 . Note that the scaling in magnitude is constant. The amplitude change of the dominant $f_{1}$ mode for the entire period 1997-2009 can also be seen from Table 6 and from the third panel of Fig. 17.

Examination of the amplitude variability of the dominant $f_{1}=64.196 \mathrm{c} \mathrm{d}^{-1}$ pulsation mode observed in 1999-2009 shows that a slow decrease of amplitude started somewhere after 1999 December whereas the rapid amplitude decrease at a rate of $\mathrm{d} m / \mathrm{d} t$ $=-0.028 \mathrm{mmag} \mathrm{d}^{-1}$ started by 2000 July (JD 2451751). Between 2000 July 25 and 2000 September 26, the pulsation semi-amplitude in $B$ changed from 7.28 to $5.1 \mathrm{mmag}$ and the corresponding $V$ amplitude from 5.87 to $4.2 \mathrm{mmag}$ (note that we used a conversion between $B$ and $V$ amplitudes and vice versa when having only one passband at our disposal). After the observational gap between 2000 October 29 and 2001 September 20 , the amplitude of the $f_{1}$ mode drastically decreased to the level of $\mathrm{A}\left(f_{1}\right)=1.02 \mathrm{mmag}$ at an approximate rate of $\mathrm{d} m / \mathrm{d} t=-0.01 \mathrm{mmag}^{-1}$. Due to the gap in our observations, the amplitude decrease may have occurred in a shorter time, thus the estimated rate is a lower limit.

During the same period, a second pulsation mode with $f_{2}=56.7 \mathrm{c} \mathrm{d}^{-1}$ appeared above the detection threshold of $0.5 \mathrm{mmag}$. The detailed analysis of individual nights from 2001 (Section 5.3) shows that around 2001 October 9 (JD 2452191) the $f_{2}$ mode increased its semi-amplitude to $2.1 \mathrm{mmag}$ (see Fig. 9); in other words, its semi-amplitude became 1.72 times larger than the semiamplitude of the formerly dominant $f_{1}$ mode.

Interestingly, the spectroscopic run by LM04, centred on the same date, confirms the amplitude inversion of the frequencies of $f_{1}^{s}=64.189(6) \mathrm{cd}^{-1}$ and $f_{2}^{s}=56.600(6) \mathrm{cd}^{-1}$ with mean pulsation amplitudes of 99(26) and 151(27) $\mathrm{ms}^{-1}$, respectively (note that the modes in LM04 have a different numbering). The RV amplitude ratio of $K\left(f_{1}\right) / K\left(f_{2}\right)=0.66(20)$ is close to the photometric amplitude ratio of $A\left(f_{1}\right) / A\left(f_{2}\right)=0.58$.

The resulting RV to $y$-band amplitude ratios for the two modes observed around JD2452191 are $2 K\left(f_{1}\right) / \Delta y\left(f_{1}\right)=$

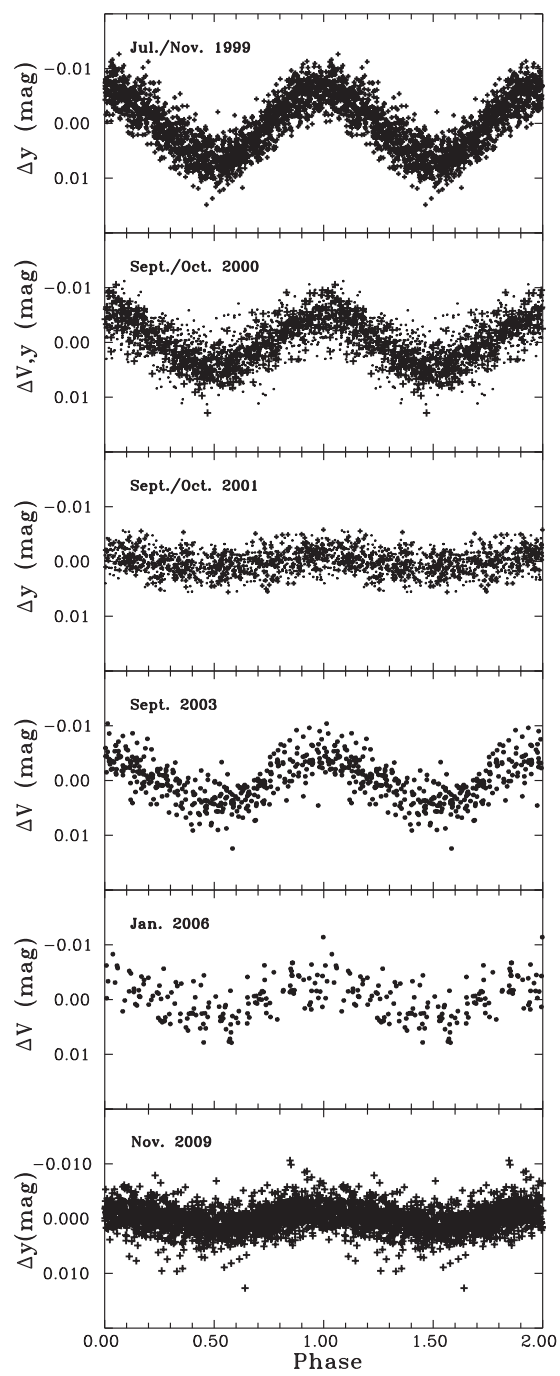

Figure 16. LCs of RZCas folded with the period of the dominant $64.193 \mathrm{c} \mathrm{d}^{-1}$ mode. From top to bottom: 1999 SNO y, 2000 SNO y (plus signs) and TSAO V (dots), 2001 SNO y (plus signs) and MLO y (dots), 2003 TSAO V, 2006 BHO V and 2009 SNO y data.

$80(21) \mathrm{km} \mathrm{s}^{-1} \mathrm{mag}^{-1}$ and $2 K\left(f_{2}\right) / \Delta y\left(f_{2}\right)=71(12) \mathrm{km} \mathrm{s}^{-1} \mathrm{mag}^{-1}$, where $K$ is the semi-amplitude in RV and $\Delta y$ the total amplitude in $y$. Both values are within the range expected for $\delta$ Scuti pulsators (e.g. Smith 1982).

As seen from Fig. 17, the amplitude of the $f_{1}$ mode slowly increased later on, at a rate of $\mathrm{d} m / \mathrm{d} t=+0.004 \mathrm{mmag} \mathrm{d}^{-1}$, from 1.02 to 2.7 mmag during the years 2002-2003. In the time span 20032006 the amplitude again decreased (note that no data were obtained in 2004 and 2005), but at a slower rate of $5 \times 10^{-4} \mathrm{mmag} \mathrm{d}^{-1}$.

The amplitude increase of the $f_{2}=56.6 \mathrm{c} \mathrm{d}^{-1}$ mode to the peak value of $2.1 \mathrm{mmag}$ in 2001 , on the other hand, was followed by its decay in course of time. It was not observed photometrically above the detection thresholds of 1.4, 0.7 and 1.0 mmag in 2002, 2003 and January 2006, respectively. But it was detected in 2009 with a semi-amplitude of $0.44 \mathrm{mmag}$. Note that the spectroscopic observations in 2006 by LM08 also found $f_{1}^{s}$ and $f_{2}^{s}$, as well as a third $f_{3}^{s}=62.406 \mathrm{c} \mathrm{d}^{-1}$ mode. These modes had RV amplitudes of 203, 94 and $145 \mathrm{~m} \mathrm{~s}^{-1}$, respectively. In 2006, the RV amplitude ratios of the $f_{1}$ and $f_{2}$ modes were $K\left(f_{1}\right) / K\left(f_{2}\right)=2.16(40)$, compared to $K\left(f_{1}\right) / K\left(f_{2}\right)=0.66(20)$ in the JD2452183-2452197 interval in 


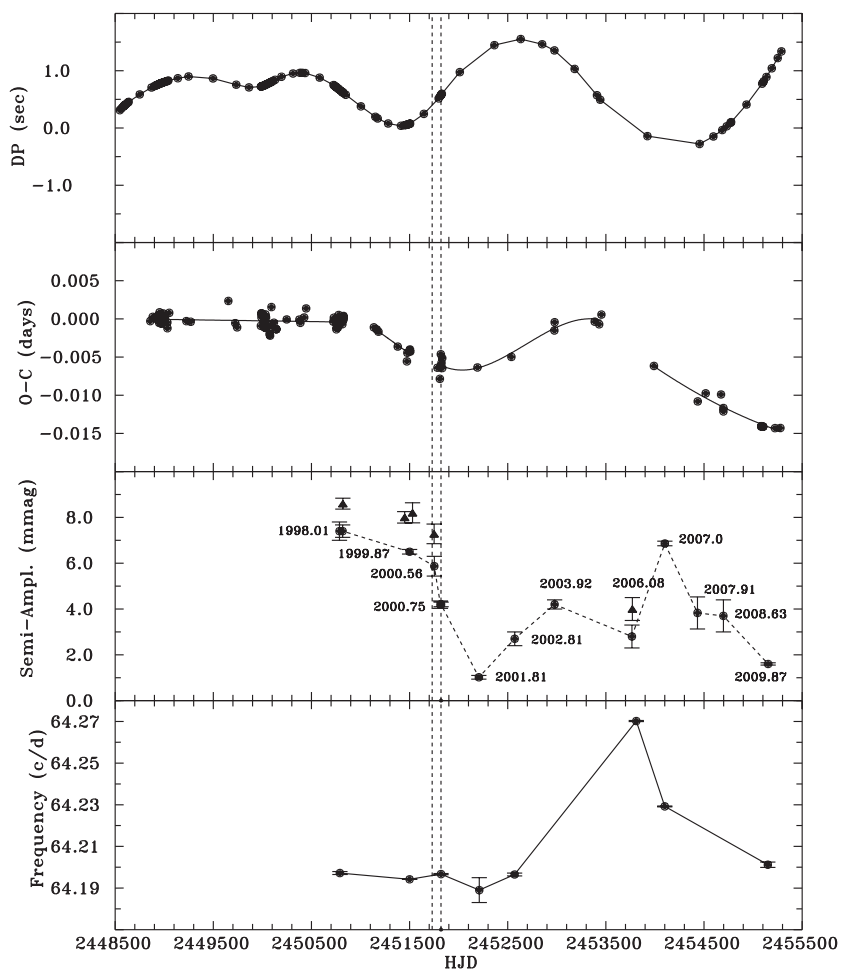

Figure 17. From top to bottom: variations of the orbital period, $O-C$ times of minima, pulsation amplitudes in the $B$ (filled triangles) and $V, y$ (filled circles) bandpasses and the frequency variations of the principal oscillation mode $f_{1}$ given in the interval from JD2448500 to JD2455100. Two vertical dashed lines show the time interval between JD 2451730 and JD 2451820 during which the $f_{1}$ mode amplitude started rapid changes.

2001. Thus, we obtained a reliable photometric detection of strong amplitude variations of all modes starting from 2000 to 2001 period that are independently confirmed by the spectroscopic observations (LM04 and LM08). Exactly 9 yr later, in 2009, the strong amplitude drop of the dominant mode $f_{1}$ occur again.

\subsection{Frequency changes}

One question still remains: Are the oscillation frequencies also unstable? The most accurate values of oscillation frequencies were found from the 1999 (Section 4.1), the re-analysis of the 2002 Soydugan et al. (2006) data, and from the 2001 (this paper and LM04) and 2006 (LM08) spectroscopic runs. These accurate frequencies will be used for further analysis of frequency variations. Frequencies and frequency deviations are presented in Table 7.

Inspection of Table 7 shows that from 1999 to 2001 the pulsation frequencies of the $f_{1}$ and $f_{2}$ modes did not vary much, but they significantly differ in 2006. In 2009, the oscillation frequency of the dominant $f_{1}$ mode returned to its previous (1999) value. The seasonal frequency variations of $f_{1}$ are shown in the bottom panel of Fig. 17. Between 2001 and 2006, frequency variations for the $f_{1}$ and $f_{2}$ modes are $\Delta f_{1}=0.0761(3) \mathrm{cd}^{-1}$ and $\Delta f_{2}=0.1655(7) \mathrm{cd}^{-1}$, respectively. These are $\Delta f_{1} / \sigma\left(f_{1}\right)=304$ and $\Delta f_{2} / \sigma\left(f_{2}\right)=301$ times larger than the measurement errors and the detection is safe.

We didn't find any frequency variation in the third photometric mode detected as $f_{3}^{p}=62.3974(5)$ in the 1999 photometric data and well detected as $f_{3}^{s}=62.40619(36)$ in the $2006 \mathrm{RV}$ data.

The frequency difference $\Delta f_{3}=0.0088(6) \mathrm{cd}^{-1}$ is $\Delta f_{3} /$ $\sigma\left(f_{3}\right)=17.6$ larger the measurement error and about 8.7 and 18.8 times less than $\Delta f_{1}$ and $\Delta f_{2}$ variations, respectively.

If the frequencies measured at different seasons indeed belong to the same modes (at the moment we keep this assumption, see further discussion and confirmation of the assumption in Section 7), then the frequency changes correspond to rates of $\left(\delta f_{1} / f_{1}\right) / \Delta T=-1.7 \times 10^{-5} \mathrm{yr}^{-1}$ and $\left(\delta f_{2} / f_{2}\right) / \Delta T=$ $-4.1 \times 10^{-4} \mathrm{yr}^{-1}$, respectively. These large negative rates are actually $2-3$ orders of magnitude larger than expected from evolutionary period changes in pre-main sequence stars that lie in the classical instability strip, and are 4-5 orders of magnitude larger than in main sequence (MS) stars (see Breger \& Pamyatnikh 1998). Thus, we claim a reliable detection of strong non-evolutionary frequency variations of yet unknown origin of two modes starting after 2001 and non-variability of the third mode. We will discuss the reason of these apparently high rates in more detail in Section 7.

\subsection{Orbital period changes}

Searching for possible correlated variations of other observables, we looked for orbital period changes of RZ Cas. The $O-C$ values for the 1992-2010 time segment are shown in the second from top panel of Fig. 17. We used the ephemeris with $T_{0}=2448960.2122$ and $P_{0}=1.1952594 \mathrm{~d}$ that fits well the 1992-2010 interval. The corresponding segment of the deviations of the local orbital period (see Fig. 2) is plotted in the top panel. As seen, after a relatively stable interval starting in early 90s, in 1998/1999 a rapid orbital period decrease of about $-0.9 \mathrm{~s}\left(\mathrm{~d} P / P=-8.71 \times 10^{-6}\right)$ occurred. This decrease was followed by a rapid increase of $+1.5 \mathrm{~s}$ $\left(\mathrm{d} P / P=1.45 \times 10^{-5}\right)$ starting in $2000 / 2001$. The cycle of the alternate negative and positive period variations was completed in 2005. It was followed by a new cycle starting with negative period changes during 2006-2009. The complete cycle length is about $9 \mathrm{yr}$, in good agreement with the $9.2 \mathrm{yr}$ orbital period variation found in our analysis in Section 3. Remarkable features visible from Fig. 17 are the rapid pulsation amplitude decrease of the principal mode $f_{1}$ in 2000/2001, followed by changes in the pulsation spectrum occurring at the time when $O-C$ values and orbital period rapidly increased. This is the first detection of nearly simultaneous changes in the orbital period and in the pulsation amplitude and pattern of the excited modes of the mass-accreting pulsating component of a binary system.

Obvious questions concern the mechanism that triggers the nearly simultaneous changes of pulsation amplitude and orbital period in-

Table 7. The $m$-dependent modal frequency variations and $m$-order identifications from PSF modelling. (p) and (s) for 2001 year columns denote the photometric and spectroscopic values of frequencies.

\begin{tabular}{|c|c|c|c|c|c|c|c|c|c|}
\hline Year & $1999(p)$ & 2001(s) & 2001(p) & $2002(p)$ & $2006(\mathrm{~s})$ & $2009(\mathrm{p})$ & $\Delta f(2006-1999)$ & $m$ & $f_{\text {cor }}(\mathrm{i})$ \\
\hline & $-1)$ & $\left(\mathrm{cd}^{-1}\right)$ & $\left(\mathrm{cd}^{-1}\right)$ & $\left(\mathrm{cd}^{-1}\right)$ & $\left(\mathrm{cd}^{-1}\right)$ & $\left(\mathrm{cd}^{-1}\right)$ & & $\left(\mathrm{cd}^{-1}\right)$ & \\
\hline$f_{1}$ & $64.19415(6)$ & $64.189(6)$ & $64.195(1)$ & $64.1965(7)$ & $64.2702(3)$ & $64.202(2)$ & $0.0761(3)$ & -1 & 63.3575 \\
\hline$f_{2}$ & $56.5955(5)$ & $56.600(4)$ & $56.605(a)$ & - & $56.7610(6)$ & $56.632(6)$ & $0.1655(7) 1$ & -2 & 54.9223 \\
\hline$f_{3}$ & $62.3974(5)$ & - & - & - & $62.4062(4)$ & - & $0.0088(6)$ & 0 & 62.3974 \\
\hline
\end{tabular}


crease. Such changes cannot be explained unless there is an interaction between the Roche lobe filling donor and the pulsating gainer star.

\section{DISCUSSION}

\subsection{The mass-transfer/accretion burst hypothesis}

A unique feature of the class of oEA stars is the simultaneous occurrence of non-stationary mass transfer, closely related to stellar evolution and non-radial oscillations of the mass gaining, primary component (Mkrtichian et al. 2002, 2004). Only this fact might explain the observed simultaneous variations in oscillation spectrum and orbital period in RZ Cas. To our knowledge, there is no observational evidence of any simultaneous orbital period increase and rapid changes of the pulsation spectrum pattern in detached binary systems with a normal $\delta$ Sct-type component. It is reasonable to suggest that the mechanism forcing rapid changes in the pulsation spectrum and the orbital period of RZ Cas are triggered by heavy mass-transfer and accretion events. The accretion hypothesis we proposed (see also Mkrtichian et al. 2003, 2005, 2007a,b) assumes that the drastic changes in the pulsation amplitudes and modal spectra of the RZCas gainer in 2000/2001 were triggered by a heavy mass-transfer/accretion burst (hereafter HMTAB) that occurred shortly before the observed changes in the pulsations. It assumed that the HMTAB itself was initiated by the magnetic activity of the secondary component.

In the next section, we discuss this hypothesis in connection with observations that self-consistently support its validity.

\subsection{Verification of the mass-transfer burst in 2000/2001 and the 2001-2009 magnetic cycle}

Here, we focus on the verification of scenario of the HMTAB in 2000/2001, using results coming from spectroscopy, photometry, celestial mechanics and asteroseismology.

\subsubsection{Light attenuation effects}

First, an HMTAB can be detected from optical effects on the observed pulsation amplitudes induced in at least two ways:

(i) Any increase in the rate of mass transfer changes the dimension and density of the gas stream, the gas-stream impact zone on the atmosphere, and the circumprimary equatorial gas 'belt' that may become optically thick. These effects can increase the amount and the asymmetry of the extinction effect across the visible surface of the pulsating gainer and are orbital phase-dependent. Gas-stream and gas-belt attenuation influences the visibility of nonradial modes, especially sectorial modes having the largest pulsation amplitude in the equatorial zone, and lead to changes in the apparent modulation of the pulsation amplitude with orbital phase. In Section 4.6, we found a direct photometric confirmation of such a modulation effect. Fig. 12 shows that the photometric amplitude variations with orbital phase show different shapes in 1999, 2000 and 2001.

(ii) Another well-observed light-attenuation effect during an HMTAB is a difference in the contributions from the 'east' and 'west' hemispheres of the gainer to the total binary flux. Just before primary eclipse, when the gas stream is seen projected on to the gainer's surface, variable attenuation by the stream leads to an asymmetry in the Rossiter effect. (iii) HMTAB and the dense circumprimary and circumbinary gas environment have attenuated the prominescence of the PSF effect during the primary eclipse phases in 2001, with particular influence on the amplitude amplification effect as discussed earlier in Section 5.2.

All attenuation effects (i-iii) were found in RZ Cas by LM04 and LM08 from comparing the 2001 with the 2006 high-resolution spectroscopic time series. Authors find clear spectroscopic signatures of a dense gas stream and circumprimary gas, and an associated, strong orbital modulation of the pulsation RV amplitude outside eclipse. They also note a strong asymmetry (anomaly) in the Rossiter effect in 2001, while it was nearly symmetrical in 2006. LM08 concluded that the mass-transfer rate in 2001 was significantly larger than in 2006. Spectroscopic modelling of the same RZ Cas spectra by Tkachenko, Lehmann \& Mkrtichian (2009) clearly confirmed the occurrence of a transient phase of rapid mass transfer in RZ Cas in 2001, while in 2006 the system was in a quiet state. Fig. 6 in LM08 shows the pulsation amplitude variations during eclipse phases in 2001 and 2006. A strong PSF effect (amplification of the apparent RV amplitude of modes) occurs during the eclipse phases in 2006 when the mass-transfer rate was low. On the other hand, the PSF effect is damped in 2001 - the season of the occurrence of HMTAB and follows the density of projected gas-stream and gas- 'belt' environment. Thus, from both spectroscopy and photometry, we find clear 'optical' evidence of higher density in the gas stream and the circumbinary environment in 2000 and 2001 compared to 1999 or 2006. This is a direct consequence of the HMTAB that started in 2000 .

\subsubsection{Mechanical effect}

HMTAB also has a direct mechanical effect on the orbital period of the binary. Conservative mass transfer from a donor to a highermass gainer gives an upper limit for an orbital period and orbital semi-axis increase of (Kwee 1958; van den Heuvel in Shore, Livio \& van den Heuvel 1994)

$$
\frac{\dot{P}}{P}=3\left(\frac{M_{1}}{M_{2}}-1\right) \frac{\dot{M}_{1}}{M_{1}}
$$

$\frac{\dot{a}}{a}=\frac{2}{3} \frac{\dot{P}}{P}$,

where $\dot{M}_{1}=-\dot{M}_{2}$ is the condition for conservative mass transfer. This calculation can only give an upper limit.

The masses of components are $M_{1}=2.056(14) \mathrm{M}_{\odot}$ (gainer) and $M_{2}=0.706(6) \mathrm{M}_{\odot}$ (donor). Inserting the orbital period increase of $1.5 \mathrm{~s}$ that started in 2000/2001 gives a mass transfer rate of $\dot{M}_{2}=+4.05 \times 10^{-6} \mathrm{M}_{\odot} \mathrm{yr}^{-1}$. We already found from orbital period variability analysis in Section 3 , that RZ Cas undergoes quasiperiodic alternate $\approx \pm 1 \mathrm{~s}$ variations of the orbital period. Thus, cyclic period variations testify cyclicity of HMTAB events in the RZ Cas system.

\subsubsection{Magnetic and flare activity in RZ Cas}

We believe that the physical reason for the increase of the masstransfer rate is the magnetic activity of the Roche lobe filling K0 III giant star (donor). Numerous radio and X-ray observations constrain magnetic and flare activity in cool Algol donors (Umana et al. 1993, Richards et al. 2003, Toscano et al. 2006) and in RZ Cas in particular 
(Drake, Simon \& Linsky 1986; Singh, Drake \& White 1995; Umana et al. 1999; Gunn \& Brady 2003; Audard, Donisan \& Güdel 2005). One rare, but strong $(\Delta m=0.5 \mathrm{mag})$ optical flare was registered in RZ Cas by Lowder $(2002,2006)$ on 1996 November 27 (JD 2450415).

In Section 3, we found from the period analysis of the orbital $(O-C)$ variations of RZ Cas, a time-scale of about 5-9 yr with an amplitude of about $\pm 1 \mathrm{~s}$. We stress that this time-scale is in well agreement with the theory of alternate orbital period changes forced by magnetic activity of cool, Roche lobe-filling components.

We think the HMTAB that caused the period increases are additive to the pure magnetically induced cyclic orbital period variations and led to shortening of the length of the cycle. While in the detached active RS CVn stars without mass-transfer events the length of the alternate cyclic variations of orbital periods by magnetic cycle is longer.

We believe that the beginning of the magnetic activity cycle is associated with the start of the orbital period decrease in 1998 and that the alternate rapid orbital period increase is associated with the occurence of HMTAB in the beginning of 2000-2001.

Duration of the recent orbital period variation cycles was about 9 yr (see Section 3.3).

We found that the next pulsation amplitude decrease occurs about 9 yr later, in 2009 (see Section 6.1 and Figs 16 and 17). The $(O-C)$ diagram conclusively shows the beginning of the next rapid orbital period decrease in 2006, about 8 yr after the 1998.

Summarizing all observational aspects discussed so far, we stress that all of them support the existence of cyclic magnetic activity of the secondary component. We conclude, in particular, that the last observed orbital period variation cycle lasting $9 \mathrm{yr}$ reflects the magnetic activity cycle of the Roche lobe filling cool companion, and this cycle caused two HMTABs, in 2001 and 2009.

\subsubsection{Asteroseismic effects}

Fourthly, the presence of HMTAB in RZ Cas is also supported by our asteroseismic results. As discussed above, we found simultaneous drastic changes of the pulsation mode amplitude spectrum and rapid orbital period changes. The difference between the oscillation frequencies measured for $f_{1}$ and $f_{2}$ in 1999 and 2006 exceeds the $300 \sigma$ detection level, while the difference in $f_{3}$ is by two orders of magnitude smaller.

The pulsation frequency depends on the mean stellar density, since $f \sim \sqrt{\rho}$ (or, in terms of radius $R$ and mass $M$, it is $f \sim M^{1 / 2} R^{-3 / 2}$ ). After a mass transfer/accretion burst, the stellar readjustment time with respect to internal structure, luminosity and mean radius is the Kelvin-Helmholtz (thermal) time-scale. For the RZ Cas parameters, it is of the order $\approx 10^{6} \mathrm{yr}$. The thermal inertia of the entire star is much too large to allow for measurable changes within a few years. Even if we assume that the star is responding to its mass increase on the dynamical time-scale for the entire star and on the thermal time-scale for its outer envelope, the amount of transferred mass $\left(\simeq 10^{-6} \mathrm{M}_{\odot}\right)$ is insufficient to produce the required mass and radii changes necessary to fit the observed frequency increase of $\left(\delta f_{1} / f_{1}\right) / \Delta T \simeq 10^{-5}-10^{-4} \mathrm{yr}^{-1}$.

In the next section, we present the mode-detuning and spinacceleration hypotheses that can give a natural explanation of the high rates of modal amplitude and frequency changes.

\subsection{Mode-detuning hypothesis}

Until now, stellar pulsation theories face difficulties in explaining why certain pulsationally unstable modes are excited with high amplitudes and others not. The selection mechanism for $\mathrm{p}$ modes is sensitive to the conditions in the stellar envelope. We assume that the mass (of the order of $10^{-6} M_{\odot}$ in the case of RZ Cas) accreted by the gainer during an HMTAB disturbs the outer envelope equilibrium and the properties of the acoustic cavity, it becomes temporarily de-tuned for the excitation of former high-amplitude modes. Thus, the mode-detuning hypothesis describes the variable excitation of modes during mass-accretion events, followed by a decay or growth of their amplitudes.

The theoretical response time of the mode selection mechanism to mass accretion is not yet modelled and unknown for different stellar models. On the other hand, the theoretical growth rates (inverse of growth times) for oscillation modes of single, non-interacting stars can be estimated from stellar models. The frequency of the principal mode in RZ Cas is $f_{1}=64.194 \mathrm{c} \mathrm{d}^{-1}(743 \mu \mathrm{Hz})$. From the pulsational constant calculated from the known radius and mass of the star, we can estimate that this frequency corresponds to the 6 th or 7 th radial overtone pulsation mode.

For early A-type MS star located inside the instability strip we get, from intermediate-mass models (e.g. table 2 in Saio \& Gautschy 2004 and fig. 5 in Handler et al. 1998), that the growth rate for a 6 th -7 th radial overtone pulsation is of the order of $10^{-7}$ $\mathrm{s}^{-1}$. Inverse of the growth rate scales of $116 \mathrm{~d}$ or roughly 4 months. From our observations, a rapid decrease of the principal pulsation amplitude occurs in 2000 on a time-scale of a few months (see Fig. 17 and discussion in Section 6.1). The principal $f_{1}$ mode amplitude increase since 2001 occurred on a time-scale of 1-2 yr. This is indeed an upper limit of amplitude growth time. We cannot fix it accurately as we do not know the duration of the mass-transfer burst in 2000-2001, it might have extended to 2002. Thus, the time-scale of growth rates might be shorter than 1-2 yr.

The observed time-scales of mode decay (and likely growth) are in good agreement with the theoretical ones and support our mode detuning hypothesis of accretion-driven changes in pulsation amplitudes.

In retrospect, the hypothesis that the mass-transfer bursts can regularly perturb the pulsation modes, forcing them to growth or/and decay, finds additional confirmation in earlier RZ Cas investigations that suggested transient pulsations in the primary component. The 1978, 1979 and 1980 observations of Olson (1982) show shortterm brightness variations of RZ Cas. The analysis of published and unpublished photoelectric observations of RZCas in 1973-1975, 1982, 1990, 1992 (Rodríguez et al. 2004a), show the absence of detectable variations or transient pulsational variations in different seasons. The periods of the suspected variability found by Rodríguez et al. (2004a) did not correspond to the principal pulsation mode at $64.195 \mathrm{c} \mathrm{d}^{-1}$ which was the dominant mode after 1997.

As an additional check, we did a DFT analysis of pulsational LCs from two nights of RZ Cas observations in 1991 obtained by Edwin \& Gears (1992). We digitized Fig. 3 of their paper, that contains four nights of CCD observations (filter is unknown) plotted versus orbital phase. We transformed the orbital phases into the JD scale using the orbital period of $1.1952594 \mathrm{~d}$. The analysis of two wellseparated (not mixed in orbital phase) nights revealed oscillation frequencies of 63.31(17) $\mathrm{c} \mathrm{d}^{-1}(A=3.1(2) \mathrm{mmag})$ and 64.27(13) $\mathrm{c} \mathrm{d}^{-1}$ ( $A=3.52(18) \mathrm{mmag}$ ) for the first and second night, respectively. While the time span and hence the frequency resolution of the digitized data is not sufficient for very accurate determination of oscillation frequencies, we conclude that oscillations at $63-64 \mathrm{c} \mathrm{d}^{-1}$ with amplitudes of about 3-3.5 mmag were excited in the 1991 season. These results independently confirm the presence of transient oscillations in the mass-accreting component of RZCas showing 
different modes in different seasons, and hence support our accretion and mode detuning hypotheses involving mass-transfer bursts.

\subsection{Spin-acceleration hypothesis and a new method of mode identification}

The second hypothesis, that we call spin-acceleration hypothesis, follows the assumptions of the mode-detuning hypothesis. Its basic assumption is that the small frequency differences observed for the $f_{1}$ and $f_{2}$ modes between 1999 and 2006 are caused by the Doppler effect affecting the NRP pulsation modes due to the acceleration of the rotation of the gainer's outer envelope. This acceleration might be caused either by the additional angular momentum accreted with the matter on the atmosphere during HMTAB or by spin-orbital coupling.

The acceleration of the gainer's outer envelope (or strong differential rotation) results in an apparent increase or decrease of the frequencies of prograde (running with the direction of rotation) or retrograde non-radial modes by an amount that is a multiple of the modal number $|m|$. Zonal modes are not affected by the Doppler effect. Thus, the Doppler effect on sectorial and tesserial modes can be effectively used for the identification of the azimuthal m-value.

Let's consider the hypothesis in detail. According to Ledoux \& Walraven (1958), the frequency in the observers frame, $f_{\text {obs }}$, is linked to the frequency in the frame corotating with the star, $f_{\text {cor }}$, as

$f_{\mathrm{obs}}^{l, m, n}=f_{\mathrm{cor}}^{l, m, n}-m f_{\mathrm{rot}}$,

where

$f_{\text {cor }}^{l, m, n}=f_{0}^{l, m, n}+m C_{n, l} f_{\text {rot }}+O\left(f_{\text {rot }}^{2}\right)$,

$f_{0}^{l, m, n}$ is the mode frequency for the non-rotating star, $f_{\text {rot }}$ is the rotation frequency, $C_{n, l}$ the rotational splitting constant and $\mathrm{O}\left(f_{\mathrm{rot}}^{2}\right)$ a second order term (Saio 1981). $l, m, n$ are, respectively, the angular degree, the azimuthal and the radial order of the non-radial modes. Note that we follow the nomenclature assigning negative and positive $m$ to prograde and retrograde modes, respectively.

The apparent frequency change by the Doppler effect due to the acceleration or braking of the gainer is

$\Delta f_{\mathrm{obs}}^{l, m, n}=-m \Delta f_{\mathrm{rot}}\left(1-C_{n, l}\right)+\Delta O\left(f_{\mathrm{rot}}^{2}\right)$.

If the acceleration of the pulsating gainer is caused by accretion of mass and angular momentum on its surface, the modal frequencies should naturally show variations due to changes of the basic stellar parameters and conditions in the outer envelope. Then we have to include the term for accretion-driven intrinsic variations of eigenmodes frequencies getting

$\Delta f_{\mathrm{obs}}^{l, m, n}=\Delta f_{0}^{l, m, n}-m \Delta f_{\mathrm{rot}}\left(1-C_{n, l}\right)+\Delta O\left(f_{\mathrm{rot}}^{2}\right)$.

However, we expect that the intrinsic frequency variations due to accretion are very small and the term $\Delta f_{0}^{l, m, n}$ can be neglected. Furthermore, the terms $m \Delta f_{\text {rot }} C_{n, l}$ and $\Delta O\left(f_{\text {rot }}^{2}\right)$ are also expected to be small, because $C_{n, l}$ is of the order of $10^{-2}$ for $n<10$ radial overtones in a $2 \mathrm{M}_{\odot}$ A-type main sequence star (Saio 1981, Shibahashi \& Saio 1985). In the result, the observed value of frequency changes due to acceleration or braking should be within few per cents of accuracy very close to $m$-times the value of the intrinsic change of the stars rotation frequency $\Delta f_{\text {rot }}$. Deviations of $\Delta f_{\text {obs }}^{l, m}$ from $m \Delta f_{\text {rot }}$ are determined by the two terms $m C_{n, l}$ and $\Delta O\left(f_{\text {rot }}^{2}\right)$ and are model and mode dependent.

Table 7 shows the frequencies and their seasonal variations for the three $f_{1}, f_{2}$ and $f_{3}$ modes. As seen, we detected a frequency increase in two modes $\left(f_{1}\right.$ and $\left.f_{2}\right)$ and no essential variations in

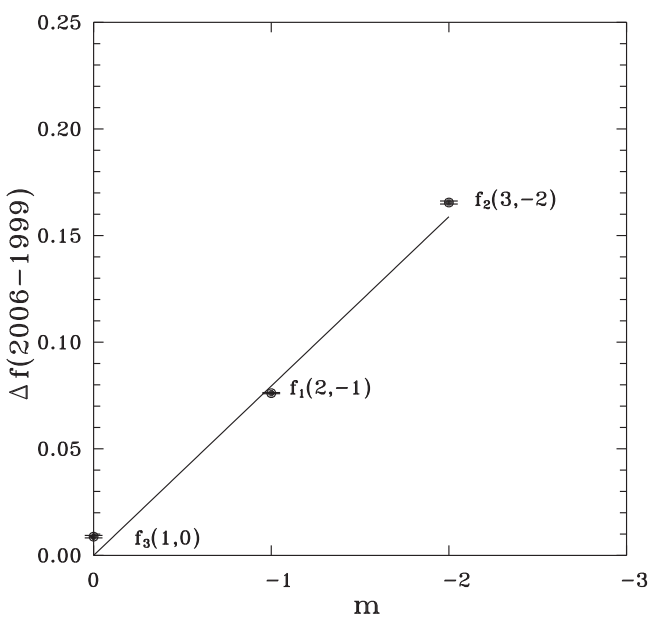

Figure 18. The modal frequency variations in 1999-2006 versus modal $m$-orders. The solid line is linking the Doppler effect $m$-dependent modal frequency variation for RZ Cas gainer, caused by rotational acceleration on $0.0794 \mathrm{~cd}^{-1}$.

the third $\left(f_{3}\right)$ one. According to our spin-acceleration hypothesis, the found frequency variations require prograde modes for $f_{1}$ and $f_{2}$ and zonal modes for $f_{3}$. The value of the frequency change $\Delta f_{1}=0.0761 \mathrm{~cd}^{-1}$ is nearly two times smaller than $\Delta f_{2}=$ $0.16551 \mathrm{c} \mathrm{d}^{-1}\left(\Delta f_{2} / \Delta f_{1}=2.17(2)\right)$. The deviation of the ratio from an integer number we relate to the errors in the frequency determinations and the contributions of the $\left(1-C_{n, l}\right)$ and $\mathrm{O}\left(f_{\mathrm{rot}}^{2}\right)$ terms. Fixing the mode identification for $f_{1}$ to $m=-1$, we immediately get $m=-2$ for the $f_{2}$ mode and $m=0$ for the $f_{3}$ mode.

Among possible $(l, m)$ identifications for $f_{2}$ mode there are $(l, m)=(2,-2)$ and $(l, m)=(3,-2)$. Note, that the first one, $l$ $=2, m=|2|$, was excluded in Section 6.1, based both on the shape (double peak) and the strength of the amplitude amplification effect. But the other one is in exact agreement with the $(l, m)=(3,-2)$ identification from the PSF method. Thus, only $l, m=2,-1$ and $l, m=3,-2$ remain for the $f_{1}$ and $f_{2}$ mode, respectively. Having safe identification of $f_{1}$ and $f_{2}$ and keeping the spin-acceleration hypothesis, we find that the mean value of $\Delta f_{1} \simeq \Delta f_{2} / 2$ is $\Delta f_{\text {rot }}=$ $\left(\Delta f_{1}+\Delta f_{2} / 2\right) / 2=0.0794 \mathrm{c} \mathrm{d}^{-1}-$ the intrinsic change of the rotational velocity of the RZ Cas gainer star between 1999 and 2006.

The bottom panel of Fig. 17 shows the dominant mode $f_{1}$ frequency variation curve for 1998-2009 caused by gainer's envelope acceleration and braking. It can be seen that the rotation frequency in 2009 returns to almost the values from 1998 to 2002. Fig. 18 shows the observed modal frequency variations versus their $m$ identifications found from the PSF modelling.

The rotation frequency of the gainer deduced from the projected equatorial velocity $v \sin i=69 \mathrm{~km} \mathrm{~s}^{-1}$ and $R=1.593$ $\pm 0.007 \mathrm{R}_{\odot}$ is $0.856 \pm \mathrm{c} \mathrm{d}^{-1}$. This value is in a good agreement with $f_{\text {rot }}=0.836 \mathrm{c} \mathrm{d}^{-1}$ following from synchronised rotation. Thus, in the interval between 1999 and 2006 the outer envelope of the RZ Cas gainer accelerated by about 9.09 per cent and until 2009 the frequency of $f_{1}$ returned close to previous values from 1999. It means that the gainer has undergone rotational braking.

We would like to stress, that we assume the acceleration of outer envelope of the gainer, but not the whole volume of the star. The latter is not realistic in terms of amount of the angular momentum transfer during HMTAB and the time-scales. Acoustic waves have a lower propagation speed in sub-photospheric layers compared to deep interiors and therefor are more sensitive to the conditions and to 
the differential rotation in the stellar outer envelope. There is still no numerical calculations of modal sensitivity to surface acceleration and there is no numerical estimations of required depths of the penetration of differential rotation to have an impact on NRPs of different $l, m$.

Finally, we want to stress again that the identifications for modal azimuthal numbers $m$ derived from the spin-acceleration hypothesis are in excellent agreement with the results coming from the eclipsemode identification, namely $l, m=2,-1, l, m=3,-2$ and $l, m=1,0$ for modes $f_{1}, f_{2}$ and $f_{3}$, respectively (see Section 6.2). Thus, these results confirm the validity of the spin-acceleration and rotationbraking hypotheses to explain the found frequency variations in RZCas. We claim the detection of new phenomena in oEA starsthe dynamical (short-term) acceleration and braking of the outer envelope of the mass-accreting component which apparently affects the NRP frequencies via the Doppler effect.

$m$-Dependent frequency variations, $m \Delta f_{\text {rot }}$, should be more prominent in higher degree modes and easily measured. This will be a future project, based on high-resolution spectra obtained in 2001 and 2006 by LM04 and LM08, respectively, and on new spectroscopic observations obtained in 2008-2016.

\subsection{A scenario for the RZ Cas activity in 1998-2009}

In the context of the HTMB hypothesis, we propose the following scenario for phenomena observed in the RZCas system between 1998 and 2009.

The magnetic activity cycle of the Roche lobe-filling K0 III donor, which began in late 1990th (approximately in 1998/1999), induced the increase in oblateness in the donor. Conservation of total angular momentum led to the period decrease of RZCas of $-0.9 \mathrm{~s}$ $\left(\dot{P} / P=-8.7110^{-6}\right)$ observed in $1998-1999$ and to a relative reduction of the orbital separation by $\dot{a} / a=2 \dot{P} / 3 P=3.8710^{-6}$ or $\Delta a=2.610^{-5} \mathrm{R}_{\odot}$ (see Table 1 for the orbital separation). This was accompanied by a proportional shrinking of the Roche lobe, the L1 point was shifted deep into the atmosphere of the KO III donor star into higher densities layers.

At this point we refer to Nazarenko, Glazunova \& Karetnikov (2001), who did a 2-D hydrodynamic simulation of mass-transfer via the L1 point using different Roche lobe overflow parameters and Kurucz's (Kurucz 1979) atmosphere models for the mass-losing star. They show, for a $\beta$ Per-type binary systems, that 1 order increase in the mass-transfer rate requires about $10^{-3}$ donor's radius shift of the L1 point into the donor's atmosphere. This is about 2 orders of values smaller than observationally estimated fast changes in the mass-transfer rate. This means, that changes in the orbital separation of components is not a determining factor in the variations of the mass-transfer rate and the dominating factor is likely the variations of the shape of the donor star.

In our estimations, the accretion rate on to the oscillating A3 V donor has been increased by 2-3 orders of magnitude and this event continued at least to 2001 September-October, when we found (see also Mkrtichian et al. 2003) a drastic decrease of photometric amplitudes and LM04 and LM08 found a safe spectroscopic confirmation of the high transfer rate.

Further, the mass transferred from donor to gainer during the burst (estimated as $2 \times 10^{-6} \mathrm{M}_{\odot}$ ) has triggered rapid changes in the mass accreted by the outer envelope of the gainer and changes in its pulsation pattern. It also forced, due to angular momentum transfer, the simultaneous rapid orbital period increase by $+1.5 \mathrm{~s}$ in $2000 / 2001$ and the increase of the orbital separation. In conse- quence, the L1 point shifted outward, to lower density layers in the atmosphere of donor star and the mass-transfer rate decreased but not to the previous levels. The HMTAB finally ended when the shape of star returns to the pre-activity state and filling of the Roche lobe. In 2006, spectroscopic observations by LM08 show no evidence of a dense gas stream or an optically thick circum-binary environment, but as we found (see Section 7.4), the RZ Cas primary component shows an accelerated outer envelope.

In 2009 , we registered a similarly drastic pulsation amplitude drop for $f_{1}$ as in 2001 and found the low-amplitude modes $f_{2}$ and $f_{4}$ modes. Remarkably, the 9-yr interval between these events is approximately the length of the suspected magnetic activity cycle found from orbital period variations in last decades. Thus, we conclude that the magnetic activity cycles, with corresponding cyclic changes of the oblateness of Roche lobe filling star and mass-transfer bursts is the most likely reason forcing the cyclic changes in the pulsation spectrum and the spin-accelerations of the mass-accreting gainer.

\section{CONCLUSIONS}

We suggest that the cyclic magnetic activity of the secondary component, yet directly not found, causes observed variations of its orbital period and triggers the transient HMTAB events in RZ Cas.

We found that HMTAB events led to simultaneous, in the orbital period and the pulsation amplitude, changes of the rich oscillation spectrum registered during the recent orbital variation cycle. All modes show strong amplitude variation over the interval of observations. We detected two rapid pulsation-amplitude decreases of the dominant mode to the level of $1 \mathrm{mmag}$ in 2001 and 2009 followed by recovery of amplitude in between of these events. We assign these mode amplitude variations to high mass-transfer and accretion episodes. We found the safe $l, m=(2,-1)$ mode identification for the principal mode using the periodic spatial filter method. We detected $m$-dependent modal frequency variations from season to season and explain them by the Doppler effect due to the acceleration and braking of rotation of the envelope of the mass-accreting component of RZ Cas. We suggest a new mode identification method for nonradial modes that utilizes the variations in the rotation of the gainer. With this method, we identified the two other dominant modes $f_{2}$ and $f_{3}$ as $l, m=(3,-2)$ and $l, m=(1,0)$ modes, respectively.

We expect a new magnetic cycle in the 2009-2018 and a new, slow amplitude increase of the dominant mode in 2010 and new cyclic accelerations of outer envelope of gainer caused by rapid mass-transfer event(s). We furthermore expect an apparent frequency variations of all detectable modes on the value of $m \Delta f_{\text {rot }}$. However, this work is out of scope of the present paper and should be investigated in the future.

We believe that other active Algol systems showing similar cyclic variations of their orbital periods and cyclic HMTA events will show similar dynamical variations in the rotation rate of the massaccreting components. If the primary components are oEA stars, the small variations in rotation period and pulsation frequencies can be measured and the identification of modes precisely found.

The new methods tested here on the RZ Cas system, namely mode identification from PSF modelling and $m$-dependent mode identification from measurements of rotation variations, have big potential for an application to extended and precise LCs of oEA stars coming from space missions. We are planning to apply these methods to LCs of the KEPLER mission stars, in particular to p-mode rich stars the KIC 8553788 and the KIC 1175495. Con- 
cerning its pulsation properties, the latter is a close twin of the RZ Cas A (Mkrtichian, in preparation).

\section{ACKNOWLEDGEMENTS}

DEM acknowledges his work as part of the research activity supported by the National Astronomical Research Institute of Thailand (NARIT), Ministry of Science and Technology of Thailand. HL acknowledges support by the DFGgrant LE 1102/3-1. ER and MGLJ acknowledge support MINECO/Spain projects AYA201454348-C3-1-R, ESP2014-54362-P and ESP2015-65712-C5-5-R. VT acknowledges support from RFBR grant no. 15-52-12371. SJ acknowledge the grant received from Indo-South Africa Science and Technology Cooperation INT/SAFR/P-3(3)2009) and NRF grant UID69828 funded by Departments of Science and Technology of the Indian and South African Governments under which the photometric observations were carried out from ARIES. KA, RK acknowledge their work as a research activity in the framework of Project No. 0075 / GF4 'Investigation of the Variability of Stars and the Processes of Substance Exchange With a Gas-Dust Disk' DEM thanks E. Soydugan for providing her 2002 observations of RZ Cas for re-analysis. MDE thanks amateur astronomer Kari Tikkanen (Oulu University) for providing us, when we were searching for evidences of orbital period jump, his visual observations, which safely showed the orbital period jump in RZ Cas in the years 2000-2001.

\section{REFERENCES}

Applegate J. H., 1992, ApJ, 385, 621

Applegate J. H., Patterson J., 1987, ApJ, 322, L99

Audard M., Donisan J. R., Güdel M., 2005, ESASP, 560, 407

Breger M., Pamyatnikh A., 1998, A\&A, 332, 958

Caton D. B., 2004, Inf. Bull. Var. Stars, 5531, 1

Christiansen J. L., Derekas A., Ashley M. C. B, Webb J. K., Hidas M. G., Hamacher D. W., Kiss L. L., 2007, MNRAS, 382, 239

Dimitrov D., Kraicheva Z., Popov V., 2008a, Inf. Bull. Var. Stars, 5842, 1

Dimitrov D., Kraicheva Z., Popov V., 2008b, Inf. Bull. Var. Stars, 5856, 1

Dimitrov D., Kraicheva Z., Popov V., 2009a, Inf. Bull. Var. Stars, 5883, 1

Dimitrov D., Kraicheva Z., Popov V., 2009b, Inf. Bull. Var. Stars, 5892, 1

Dimitrov D., Kraicheva Z., Popov V., Genkov V., 2010, Inf. Bull. Var. Stars, 5925,1

Drake S. A., Simon T., Linsky J. L., 1986, AJ, 91, 1229

Dvorak S., 2009, CoAst, 160, 64

Dziembowski W., 1977, Acta Astron., 27, 203

Edwin R. P., Gears R. T., 1992, PASP, 104, 1234

Foster G., 1996, AJ, 112, 1709

Gamarova A. Yu., Mkrtichian D. E., Rodríguez E., Costa V., López-González M. J., 2003, PASPC, 292, 369

Grossman A., Morlet J., 1984, SIAM J. Math. Anal., 15, 723

Gunn A. G., Brady P. A., 2003, MNRAS, 346, 337

Gunsriwiwat K., Mkrtichian D. E., 2015, Inf. Bull. Var. Stars, 6148, 1

Gunsriwiwat K., Mkrtichian D. E., 2016, Inf. Bull. Var. Stars, 6178, 1

Hambsch F.-J., Lampens P., van Cauteren P., Kleidis S., Robertson C. W., Krajci T., Wils P., 2010, Inf. Bull. Var. Stars, 5949, 1

Handler G., Pamyatnykh A. A., Zima W., Sullivan D. J., Audard N., Nitta A., 1998, MNRAS, 295, 377

Hegedüs T., Szatmáry K., Vinkó K., 1992, Ap\&SS, 187, 57

Jeon Y.-B., Kim S.-L., Lee M. G., Lee H., Lee J. W., 2006, ApJ, 636, L129

Kim S.-L., Lee J. W., Kwon S.-G., Youn J.-H., Mkrtichian D. E., Kim C., 2003, A\&A, 405, 231

Kim S.-L., Koo J. R., Lee J. A., Kang Y. B., Choo K. J., Mkrtichian D. E., Kim S.-H., Lee D. J., Lee J. W., 2004a, Inf. Bull. Var. Stars, 5537, 1

Kim S.-L., Kang Y. B., Koo J. R., Mkrtichian D. E., Lee J. W., 2004b, Inf. Bull. Var. Stars, 5538, 1
Kim S.-L., Lee J. W., Kang Y. B., Koo J.-R., Mkrtichian D. E., 2005a, Inf. Bull. Var. Stars, 5628, 1

Kim S.-L., Lee J. W., Koo J.-R., Kang Y. B., Mkrtichian D. E., 2005b, Inf. Bull. Var. Stars, 5629, 1

Kim S.-L., Lee J. W., Koo J.-R., Kang Y. B., Lee J. W., Mkrtichian D. E., 2005c, Inf. Bull. Var. Stars, 5669, 1

Kreiner J. M., 2004, Acta Astron., 54, 207

Kurucz R. L., 1979, ApJS, 40, 1

Kusakin A. V., Mkrtichian D. E., Gamarova A. Y., 2001, Inf. Bull. Var. Stars, 5106,1

Kwee K. K., 1958, BAN, 14, 131

Lacorte M. B., van Hamme W., 1999, IAPPP, 76, 22

Lampens P., Van Cauteren P., Strigachev A., Kim S.-L., Kang Y. B., Koo J.-R., Mkrtichian D. E., 2004, Inf. Bull. Var. Stars, 5572, 1

Lampens P. et al., 2011, A\&A, 534, A111

Lanza A. F., 2006, MNRAS, 369, 1773

Lanza A. F., Rodono M., 1999, A\&A, 349, 887

Lanza A. F., Rodono M., 2004, AN, 325, 393

Ledoux P., Walraven T., 1958, Handbuch der Phys., 51, 353

Lee J. W., Kim S.-L., Hong K., Koo J.-R., Lee C.-U., Youn J.-H., 2016a, AJ, 151, 25

Lee J. W., Hong K., Kim S.-L., Koo J.-R., 2016b, MNRAS, 460, 4220

Lehmann H., Mkrtichian D., 2004, A\&A, 413, 293 (LM04)

Lehmann H., Mkrtichian D., 2008, A\&A, 480, 247 (LM08)

Lehmann H., Southworth J., Tkachenko A., Pavlovski K., 2013, A\&A, 557, 79

Lenz P., Breger M., 2005, Commun. Asteroseismol., 146, 53

Liakos A., Niarchos P., 2009, CoAstr., 160, 2

Liakos A., Cagaš P., 2014, Ap\&SS, 353, 559

Liakos A., Niarchos P., 2013, Ap\&SS, 343, 123

Liakos A., Niarchos P., Soydugan E., Zasche P., 2012, MNRAS, 422, 1250

Lombard F., Koen C., 1993, MNRAS, 263, 309

Lowder W. M., 2006, JAAVSO, 35, 182

Matese J. J., Whitmire D. P., 1983, A\&A, 117, L7

Maxteed P. F. L., Hill G., Hilditch R. W., 1994, A\&A, 282, 821

Middleton C. T., 2011, MNSSA, 70, 189

Mkrtichian D. E., 1992, Stellar Magnetism, Nauka, Sankt Petersburg, 260

Mkrtichian D. E., 1993, PhD Thesis, Odessa State Univ., Odessa

Mkrtichian D. E., Gamarova A. Y., 2000, Inf. Bull. Var. Stars, 4836, 1

Mkrtichian D. E., Kusakin A. V., Gamarova A. Y., Nazarenko V., 2002, PASPC, 259, 96

Mkrtichian D. E. et al., 2003, PASPC, 292, 113

Mkrtichian D. E. et al., 2004, A\&A, 419, 1015

Mkrtichian D. E., Rodríguez E., Olson E. C., Kusakin A. V., Kim S.-L., Lehmann H., Gamarova A. Y., Kang Y. W., 2005, PASPC, 333, 197

Mkrtichian D. E. et al., 2006, Ap\&SS, 304, 169

Mkrtichian D. E. et al., 2007a, PASPC, 370, 194

Mkrtichian D. E. et al., 2007b, in Kang Y.-W., Soonthornthum B., eds, Proc. of the First Thailand-Korea Joint Workshop on Stellar Astrophysics, Chiang-Mai Univ., Chiang-Mai, Thailand, p. 49

Mkrtichian D. E., Gunsriwiwat K., Komonjinda S., 2016, Inf. Bull. Var. Stars, 6182, 1

Mkrtichian D. E., Athano N., Awipan S., 2017a, Inf. Bull. Var. Stars, 6210, 1

Mkrtichian D. E. et al., 2017b, Inf. Bull. Var. Stars, 6221, 1

Narusawa S.-Y., Ozaki S., Sadakane K., 2006, PASPJ, 58, 617

Nather R. E., Robinson E. L., 1974, ApJ, 190, 637

Nazarenko V., Glazunova L., Karetnikov V. G., 2001, Astron. Rep., 45, 452

Norton A. J., Lohr M. E., Smalley B., Wheatley P. J., West R. G., 2016, A\&A, 587, A54

Ohshima O., Narusawa S.-Y., Akazawa H., Fujii M., Kawabata T., Ohkura N., 1998, Inf. Bull. Var. Stars, 4581, 1

Ohshima O., Narusawa S.-Y., Akazawa H., Arai K., Fujii M., Kawabata T., Ohkura N., 2001, AJ, 122, 418

Olson E. C., 1982, ApJ, 259, 702

Pigulski A. G., Michalska G., 2007, Acta Astron., 57, 61

Richards M. T. et al., 2003, ApJ, 147, 337

Rodríguez E. et al., 2004a, MNRAS, 347, 1317 
Rodríguez E., García J. M., Gamarova A. Y., Costa V. DaszynskaDaszkiewicz J., López-González M. J., Mkrtichian D. E., Rolland A., 2004b, MNRAS, 353, 310

Rodríguez E. et al., 2010, MNRAS, 408, 2149

Rüdiger G., Elstner D., Lanza A. F., Granzer Th., 2002, A\&A, 392, 605

Saio H., 1981, ApJ, 244, 299

Saio H., Gautschy A., 2004, MNRAS, 350, 485

Shibahashi H., Saio H., 1985, PASJ, 37, 245

Shore S. N., Livio M., van den Heuvel E. P. J., 1994, in Nussbaumer H., Orr A., eds, Interacting Binaries, Lecture Notes of the 22nd Advanced Course of the SSAA. Springer-Verlag, Berlin, Heidelberg

Singh K. P., Drake S. A., White N. E., 1995, ApJ, 445, 840

Smith M., 1982, ApJ, 254, 242

Soydugan E., Soydugan F., Ibanoglu C., Frasca A., Demircan O., Akan M. C., 2006, AN, 327, 905

Soydugan E. et al., 2008, CoAst, 157, 379

Soydugan E., Soydugan F., Senyuz T., Puskullu C., Tuysus M., Bakis V., Bilir S., Demircan O., 2009, Inf. Bull. Var. Stars, 5902, 1

Soydugan F., Soydugan E., Kanvermez C., Liakos A., 2013, MNRAS, 432, 3278

Sumter G. C., Beaky M. M., 2007, Inf. Bull. Var. Stars, 5798, 1

Tkachenko A., Lehmann H., Mkrtichian D. E., 2009, A\&A, 504, 991

Toscano S., Buemi C., Cerrigone L., Leto P., Rodono M., Trigilio C., Umana G., 2006, Mem S. A. It. Suppl., 9, 276
Turcu V., Pop A., Moldovan D., 2008, Inf. Bull. Var. Stars, 5826, 1

Turner V., 2011, MS Thesis, Ball State Univ.

Umana G., Trigilio R. M., Hjellming S., Catalano S., Rodono M., 1993, A\&A, 267, 126

Umana G., Leto P., Trigilio C., Hjellming R. M., Catalano S., 1999, A\&A, 342, 709

Unno W., Kiguchi M., Kitamura M., 1994, PASJ, 46, 613

Wilson R. E., Van Hamme W., 2009, ApJ, 699, 118

Yang Y.-G., Wei J.-Y., Li H.-L., 2014, AJ, 147, 35

Zhang X. B. et al., 2014, AJ, 148, 106

Zhang X. B., Luo Y. P., Wang K., Luo C. Q., 2015, AJ, 150, 37

\section{SUPPORTING INFORMATION}

Supplementary data are available at MNRAS online.

Please note: Oxford University Press is not responsible for the content or functionality of any supporting materials supplied by the authors. Any queries (other than missing material) should be directed to the corresponding author for the article.

\section{APPENDIX A: ADDITIONAL TABLES}

Table A1. The up-to-date list of oEA stars. The pulsation frequency $F_{\text {puls }}$ is given for the dominant mode.

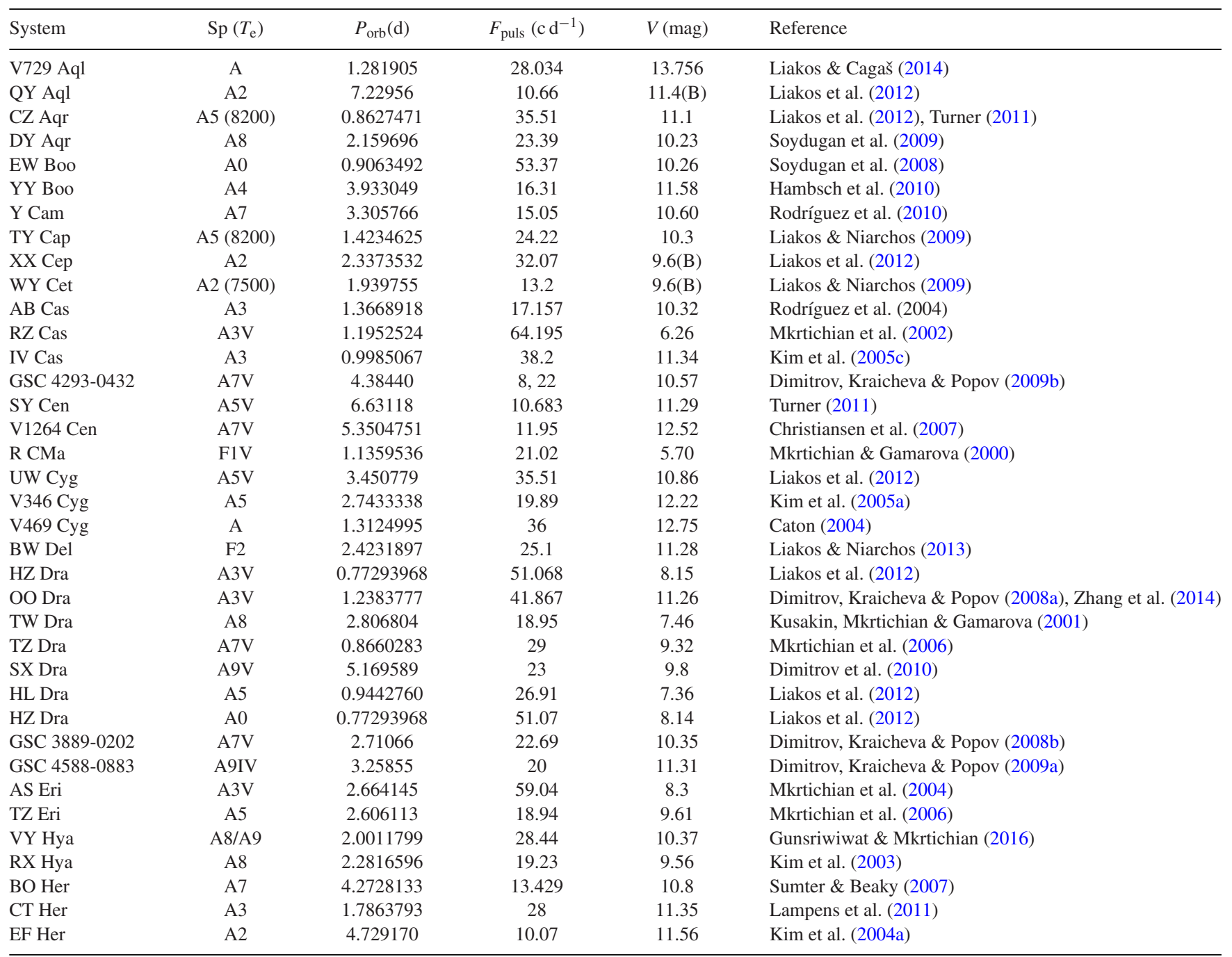


Table A1. - continued

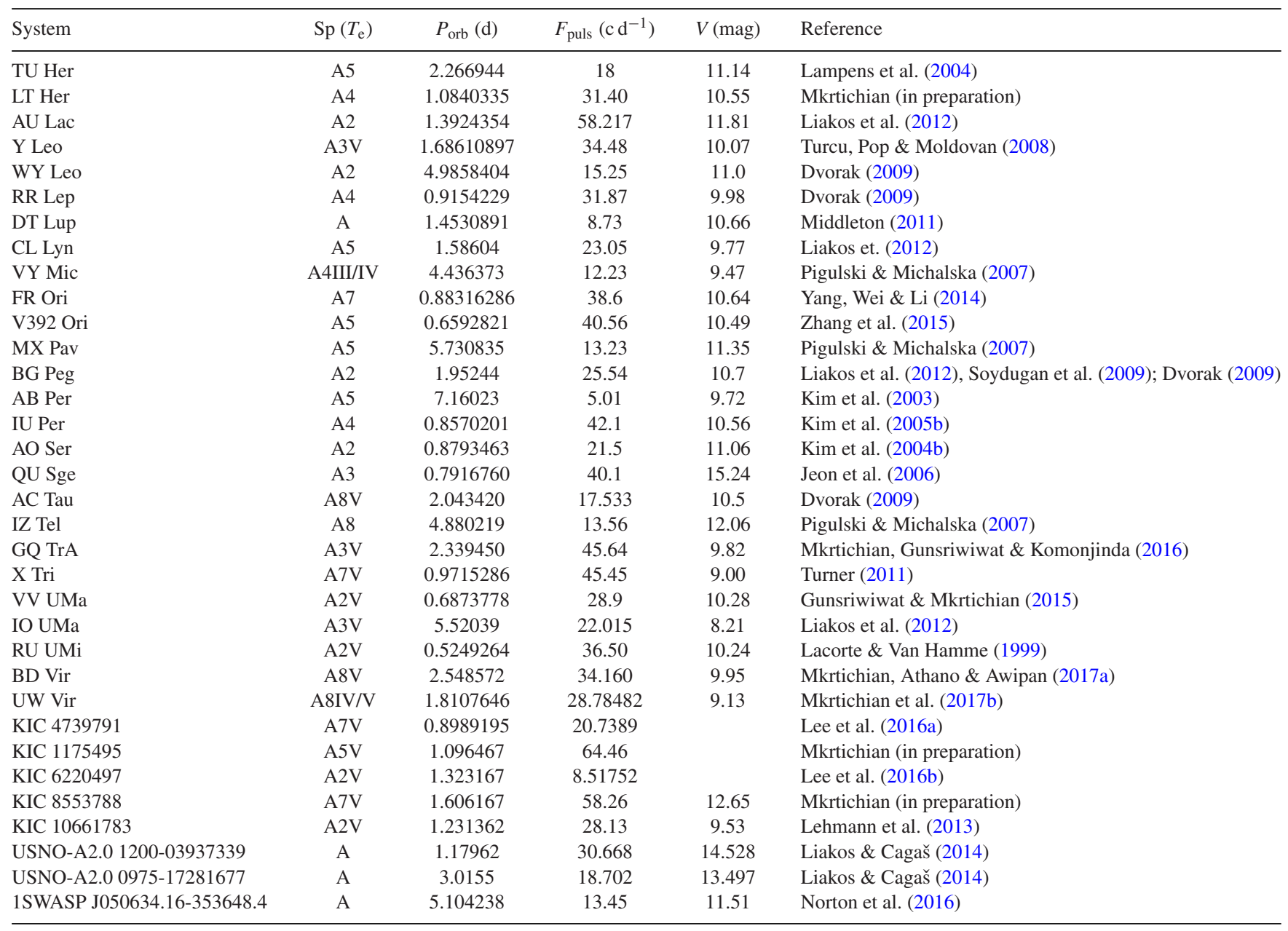

Table A2. Observatories and facilities participating in the 1999-2009 photometric observations of RZCas.

\begin{tabular}{|c|c|c|c|}
\hline Observatory & Tel./phot. & Filters & Comp/check stars \\
\hline Mount Lemmon Optical Astronomy Observatory (MLOAO), USA & $1.0 \mathrm{~m} / \mathrm{CCD}$ & $V, B$ & $\mathrm{C} 7, \mathrm{C} 8, \mathrm{C} 11, \mathrm{C} 9$ \\
\hline Odessa Astronomical Observatory (OAO) & $0.5 \mathrm{~m} / \mathrm{CCD} / \mathrm{PMT}$ & V & $\mathrm{C} 1, \mathrm{C} 3$ \\
\hline Abastumani Astrophysical Observatory (AAO), Georgia & 1.25 m/PMT & $b$ & $\mathrm{C} 10$ \\
\hline Sobaeksan Optical Astronomy Observatory (SOAO), Korea & $0.6 \mathrm{~m} / \mathrm{CCD}$ & $V$ & $\mathrm{C} 1, \mathrm{C} 3$ \\
\hline Cryonery Observatory $(\mathrm{CO})$, Greece & $1.2 \mathrm{~m} / \mathrm{CCD}$ & $V$ & HD 15948 \\
\hline Maydanak Observatory (MO), Uzbekistan & $0.6 \mathrm{~m} / \mathrm{PMT}$ & $B, V$ & $\mathrm{C} 1, \mathrm{C} 3$ \\
\hline Beercel Hills Observatory (BHO), Belgium & $0.25 \mathrm{~m}, 0.4 \mathrm{~m} / \mathrm{CCD}$ & $V$ & $\mathrm{C} 1, \mathrm{C} 4, \mathrm{C} 5$ \\
\hline Tien Shan Observatory (TSAO), Kazakhstan & $0.5 \mathrm{~m} / \mathrm{PMT}$ & $B, V$ & $\mathrm{C} 1 \mathrm{C} 3$ \\
\hline Private Observatory (POB), Brno, Czech Republic & $3.5 \mathrm{~cm} / \mathrm{CCD}$ & $V$ & \\
\hline Private Observatory (POJP), Jílové u Prahy, Czech Republic & $3.4 \mathrm{~cm} / \mathrm{CCD}$ & $V$ & \\
\hline
\end{tabular}

Notes. $\mathrm{C} 1=\mathrm{SAO} 12408, \mathrm{C} 2=\mathrm{SAO} 12345, \mathrm{C} 3=\mathrm{SAO} 12386, \mathrm{C} 4=\mathrm{SAO} 12411, \mathrm{C} 5=\mathrm{SAO} 12413, \mathrm{C} 6=\mathrm{SAO} 12421, \mathrm{C} 7=\mathrm{SAO} 12458, \mathrm{C} 8=\mathrm{GSC}$ 04317-01496, C9 = GSC04317-01390, C10 = TYC 4317-1437-1 
Table A3. Log of 1999-2009 photometric observations of RZ Cas (see Table A2 for the abbreviations of observatories).

\begin{tabular}{|c|c|c|c|c|c|c|c|}
\hline Date & HJD $2450000+$ & Filter & Obs. & Date & HJD $2450000+$ & Filter & Obs. \\
\hline 24.09 .99 & $1446.3096-.5591$ & $b$ & $\mathrm{AAO}$ & 20.09 .01 & 2172.5560-.6917 & uvby & SNO \\
\hline 26.09 .99 & $1448.3458-.3999$ & $b$ & $\mathrm{AAO}$ & 02.10 .01 & $2184.5464-.6890$ & uvby & SNO \\
\hline 27.09 .99 & $1449.3069-.5025$ & $b$ & AAO & 05.10 .01 & $2188.0080-.1667$ & V & SOAO \\
\hline 01.10 .99 & $1453.3719-.5820$ & $b$ & AAO & 06.10 .01 & $2189.0033-.2470$ & $V$ & SOAO \\
\hline 04.10 .99 & $1456.4034-.5777$ & $b$ & $\mathrm{AAO}$ & 08.10 .01 & $2190.9842-1.120$ & V & SOAO \\
\hline 10.12 .99 & $1523.4088-.5940$ & $b$ & $\mathrm{AAO}$ & 09.10 .01 & $2191.5706-.7057$ & $u v b y$ & SNO \\
\hline 14.12 .99 & $1527.0711-.4499$ & $B$ & TSAO & 24.10 .01 & 2206.7037-.9578 & $y$ & MLO \\
\hline 15.12 .99 & $1528.0701-.2610$ & $B$ & TSAO & 26.10 .01 & $2208.6592-.7917$ & $y$ & MLO \\
\hline 16.12 .99 & $1529.1495-.2799$ & $B$ & TSAO & 01.12 .03 & $2975.0803-.3377$ & V & TSAO \\
\hline 25.07 .00 & $1751.3765-.4535$ & $B$ & MO & 03.12 .03 & $2977.2620-.5369$ & $V$ & TSAO \\
\hline 18.09 .00 & $1806.2130-.4535$ & V & TSAO & 04.12 .03 & $2978.0740-.3854$ & V & TSAO \\
\hline 20.09 .00 & 1808.6146-.6972 & uvby & SNO & 23.01 .06 & $3759.2551-.4462$ & V & $\mathrm{BHO}$ \\
\hline 29.09 .00 & $1817.0164-.2591$ & V & SOAO & 30.12 .06 & $4100.1031-.2392$ & $B$ & ARIES \\
\hline 30.09 .00 & 1818.0239-.2906 & $V$ & SOAO & 02.01 .07 & $4103.0835-.2273$ & $B$ & ARIES \\
\hline 03.10 .00 & $1821.0268-.2716$ & $V$ & SOAO & 05.01 .07 & 4106.0644-.1928 & $B$ & ARIES \\
\hline 04.10 .00 & $1822.0081-.1395$ & $V$ & SOAO & 28.11 .07 & $4433.0321-.1834$ & V & SOAO \\
\hline 05.10 .00 & 1822.9954-3.095 & $V$ & SOAO & 29.11 .07 & $4434.1290-.2086$ & $V$ & SOAO \\
\hline 06.10 .00 & $1824.0571-.2105$ & $V$ & SOAO & 30.11 .07 & $4434.9533-.2302$ & V & SOAO \\
\hline 07.10 .00 & $1825.0869-.3607$ & $V$ & SOAO & 18.08 .08 & $4697.5448-.6218$ & V & Private obs. \\
\hline 14.10 .00 & $1832.2340-.6399$ & $V$ & SOAO & 10.11 .09 & $5146.2911-.7312$ & $u, v, b, y$ & SNO \\
\hline 14.10 .00 & $1832.2624-.5055$ & V & $\mathrm{CO}$ & 11.11 .09 & $5147.2913-.7308$ & $u, v, b, y$ & SNO \\
\hline 16.10 .00 & $1834.2896-.4106$ & uvby & SNO & 12.11.09 & $5148.3507-.7328$ & $u, v, b, y$ & SNO \\
\hline 17.10 .00 & $1835.2307-.3160$ & V & TSAO & 22.11 .09 & $5158.5598-.7398$ & $u, v, b, y$ & SNO \\
\hline 17.10 .00 & $1835.2870-.3602$ & uvby & SNO & 23.11 .09 & 5159.4767-.7406 & $u, v, b, y$ & SNO \\
\hline
\end{tabular}

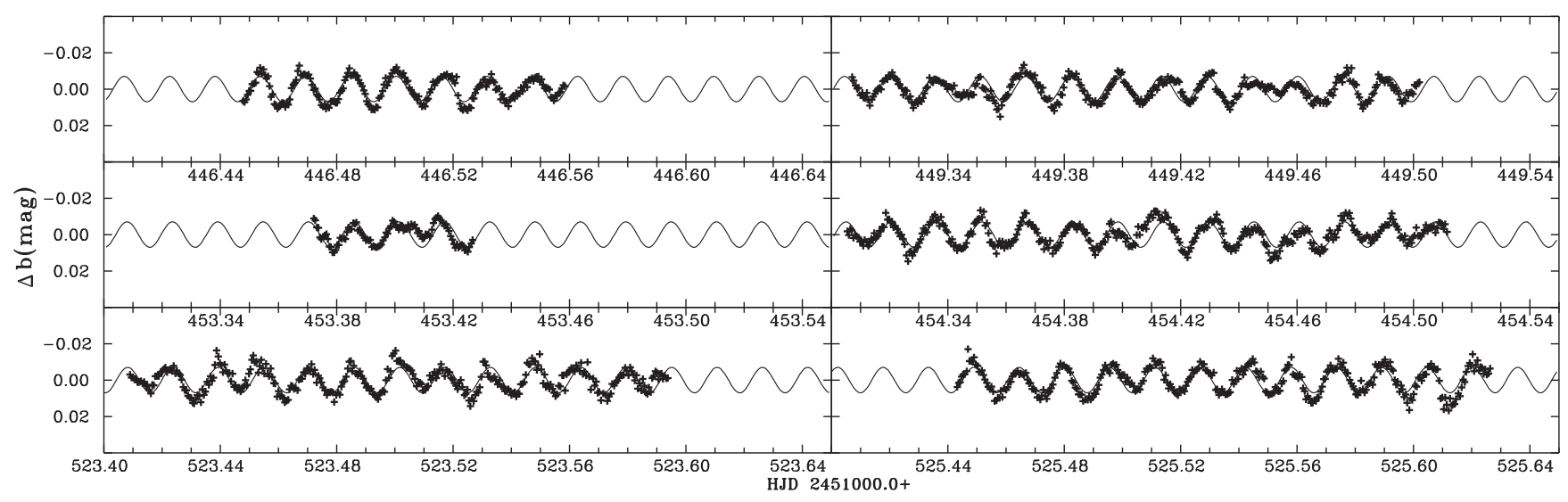

Figure B1. AAO 1999 b-filter data obtained with the $1.25 \mathrm{~m}$ telescope at the Abastumani Astrophysical Observatory (Georgia) 


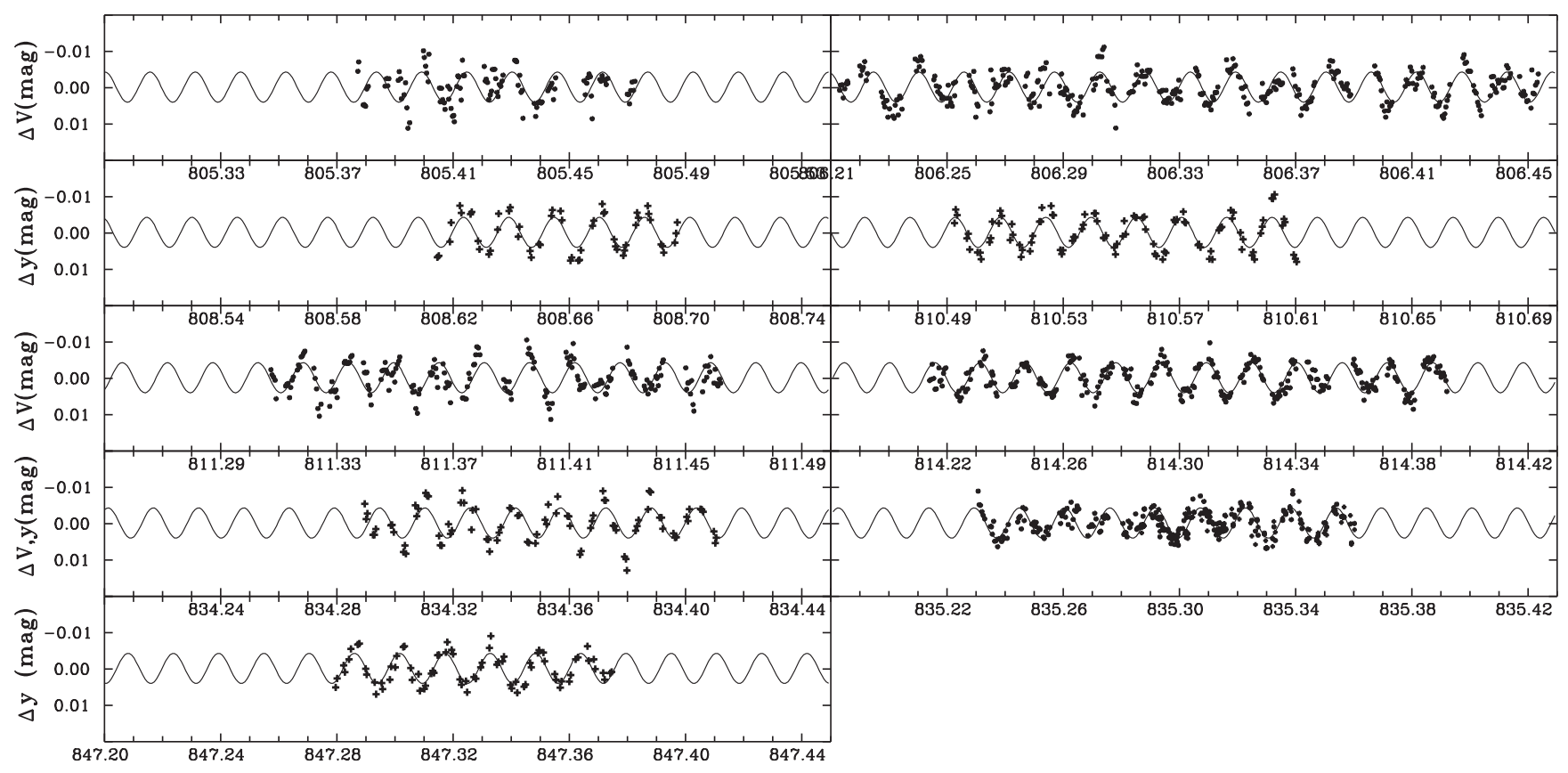

Figure B2. The pulsation Strömgren $y$ and Johnson V light curves of RZ Cas observed in 2000.

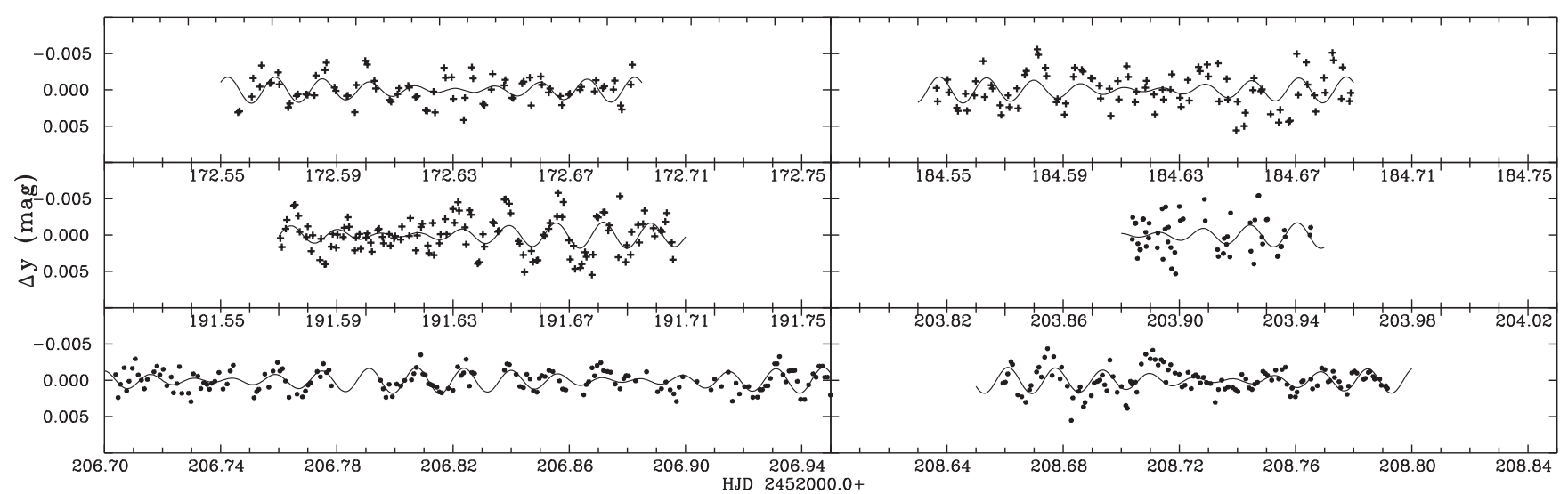

Figure B3. Strömgren $y$ light-curve oscillation observed in 2001 .

${ }^{1}$ National Astronomical Research Institue of Thailand, 260 Moo 4, T. Donkaew, A. Maerim, Chiangmai, 50180 Thailand

${ }^{2}$ Thüringer Landessternwarte Tautenburg, Sternwarte 5, D-07778 Tautenburg, Germany

${ }^{3}$ Instituto de Astrofísica de Andalucía, CSIC, PO Box 3004, E-18080 Granada, Spain

${ }^{4}$ Departmment of Astronomy, University of Illinois, 1002 West Green Street, Urbana, IL 61801-3074, USA

${ }^{5}$ Korea Astronomy and Space Science Institute, Daejeon, 34055, Korea

${ }^{6}$ Fesenkov Astrophysical Institute, Observatory street 23, 050020, Almaty, Kazakhstan

${ }^{7}$ National Center of Space Researches and Technologies, Shevchenko str. 15, Almaty, 050010 Kazakhstan

${ }^{8}$ National Youth Space Center, Jeollanam-do 59567, Korea

${ }^{9}$ E. K. Kharadze Abastumani Astrophysical Observatory, Ilia State University, 0162, Tbilisi, Georgia

${ }^{10}$ Siddhi Vinayak Engineering \& Management College Alwar, Alwar 301001, Rajasthan, India

${ }^{11}$ Aryabhatta Research Institute of Observational Sciences (ARIES), Manora peak, Nainital 263002, India

${ }^{12}$ Koninklijke Sterrenwacht van België, Ringlaan 3, B-1180 Brussel, Belgium
${ }^{13}$ Humain station, Koninklijke Sterrenwacht van België, Ringlaan 3, B-1180 Brussel, Belgium

${ }^{14}$ Odessa National Academy of Telecommunications, Kuznechnaya street 1 , Odessa 65029, Ukraine

${ }^{15}$ Astronomical Observatory, Odessa National University, Shevchenko Park, Odessa 650014, Ukraine

${ }^{16}$ Crimean Astrophysical Observatory, Nauchny, Crimea, 98409 Ukraine

${ }^{17}$ Department of Astrophys, Astronomy \& Mechanics, Athens University, Panepistimiopolis, Zografos, Athens 15784, Greece

${ }^{18}$ Private Observatory, Výpustky 5, Brno, CZ-614 00, Czech Republic

${ }^{19}$ Private Observatory, Pohori 71, Jilove u Prahu,CZ-254 01, Czech Republic

${ }^{20}$ Crimean Federal University, 295007, Vernadsky av. 4, Simferopol, Crimea

${ }^{21}$ National Nanotechnology Open Laboratory, Al Farabi Kazakh National University, Al Farabi Av., 71, Almaty 050038, Kazakhstan

${ }^{22}$ Department of Astronomy and Space Science, Sejong University, Seoul, 143-747, Korea

This paper has been typeset from a $\mathrm{T}_{\mathrm{E}} \mathrm{X} / \mathrm{L} \mathrm{T} \mathrm{E} \mathrm{X}$ file prepared by the author. 